\title{
A new displacement-based framework for non-local Timoshenko beams
}

\author{
Giuseppe Failla $\cdot$ Alba Sofi $\cdot$ Massimiliano Zingales
}

Received: 22 April 2014/Accepted: 4 March 2015/Published online: 22 March 2015

(C) Springer Science+Business Media Dordrecht 2015

\begin{abstract}
In this paper, a new theoretical framework is presented for modeling non-locality in shear deformable beams. The driving idea is to represent non-local effects as long-range volume forces and moments, exchanged by non-adjacent beam segments as a result of their relative motion described in terms of pure deformation modes of the beam. The use of these generalized measures of relative motion allows constructing an equivalent mechanical model of non-local effects. Specifically, long-range volume forces and moments are associated with three spring-like connections acting in parallel between couples of nonadjacent beam segments, and separately accounting
\end{abstract}

G. Failla · A. Sofi $(\bowtie)$

Dipartimento di Ingegneria Civile, dell'Energia, dell'Ambiente e dei Materiali (DICEAM), Università "Mediterranea" di Reggio Calabria, Via Graziella, Località Feo di Vito, 89124 Reggio Calabria, Italy e-mail: alba.sofi@unirc.it

G. Failla

e-mail: giuseppe.failla@unirc.it

\section{Zingales}

Dipartimento di Ingegneria Civile, Ambientale ed Aerospaziale (DICA), Università di Palermo, Viale delle Scienze ed.8, 90128 Palermo, Italy

e-mail: massimiliano.zingales@unipa.it;

massimiliano.zingales@iemest.eu

\section{Zingales}

Dipartimento di Biomeccanica e Biomateriali, Istituto Euro-Mediterraneo di Scienza e Tecnologia, I.E.Me.S.T., Via Emerico Amari n.128, 90100 Palermo, Italy for pure axial, pure bending and pure shear deformation modes. The variational consistency of the proposed non-local beam model is demonstrated by minimization of an appropriate total potential energy functional. Numerical results concerning the static behavior for different boundary and loading conditions are presented. It is shown that the proposed nonlocal beam model is able to capture experimental data on the static deflection of micro-beams, available in the literature.

Keywords Non-local Timoshenko beam · Longrange interactions $\cdot$ Pure deformation modes $\cdot$ Springlike connections

\section{Introduction}

It is now well understood that a classical local continuum modeling, although certainly accurate in a wide spectrum of engineering applications, may fail to capture those phenomena where microstructure and long-range intermolecular forces play a crucial role. Such inadequacy, due to the intrinsic free scale formulation of the classical local continuum theory, has been revealed theoretically based on molecular simulations, and experimentally by static and dynamic tests on several materials.

Molecular simulations may seem a most appropriate way to account for microstructural effects, but they 
involve as a major drawback a considerable computational effort. For this reason, and in recognition of the fact that even to build a molecular model some theoretical assumptions are still needed, scientists and engineers have turned their attention to the formulation of "enriched" continua, i.e. classical continuum models where microstructural effects are accounted for in an average sense, by introducing appropriate non-local terms. Since the pioneering work by Eringen $[1,2]$, these formulations have been awarded a considerable attention, especially due to the fact that a continuum formulation, although endowed with the additional non-local terms, generally allows established numerical solution methods to be applied, with considerable advantages for design purposes. In this context the following approaches can be cast: the wellknown Eringen's integral theory [1, 2], involving a stress-strain relation between the stress at a given point and the strain in the whole volume of the continuum; the gradient elasticity theories [3, 4], with constitutive equations depending on the gradients of stresses or strains; the peridynamic theory [5], involving long-range elementary forces depending on relative displacements between non-adjacent points; the well-known micropolar "Cosserat" theory [6] and the couple-stress theory [7], according to which any material point is endowed with translational and rotational degrees of freedom, with resulting workconjugate curvatures and couple stresses. Regarding micropolar or couple-stress theories, many interesting studies have been devoted to explain, on a physical basis, the relation with microstructural effects, see for instance those by Kröner [8] and Lakes [9].

Within the context of non-local enriched continua formulations, several non-local beam theories have been derived. The interest in non-local beam models is certainly motivated by the increasing importance of small-size beam-like devices, used for instance as sensors or actuators in micro- and nano-technologies (see the comprehensive review by Qian et al. [10]), where a significant deviation from the theoretical predictions of the classical Euler-Bernoulli (EB) or Timoshenko (TM) beam theory has been revealed by atomistic simulations [11] and experimental evidence on several materials, such as epoxy [12], polypropylene [13], graphite [14] and copper [15]. Existing nonlocal beam models have generally involved linearlyelastic non-local terms, used in conjunction with the classical continuum of the EB or TM beam theories. Dynamic and static responses have been investigated.

Most of the early non-local beam models have been built on Eringen's integral theory [1, 2]. In this context, based on the assumption that non-local terms affect the normal stress only, the motion equation of a EB beam has been formulated by Zhang et al. [16], Xu [17], Wang and Varadan [18]. Wang and Varadan [18] have also formulated the motion equations of a TM beam. Lu and et al. have later clarified some inconsistencies in these first studies, due to an incorrect use of Eringen's non-local stress law [19]. Following these clarifications, Reddy [20] and Aydogdu [21] have formulated alternative higher-order non-local beam theories based on certain assumptions on the displacement field.

There exist also many non-local beam models derived from non-local theories alternative to Eringen's integral theory. For instance, non-local EB beam models have been built by Kong et al. [22] based on a modified couple stress theory, by Zhang et al. [23] based on a so-called hybrid approach, which involves a strain energy functional depending on local and nonlocal curvatures. A non-local TM beam model has been built by Wang et al. [24] in conjunction with the gradient elasticity theory presented by Lam et al. [12], and by Ma et al. [25] based on a modified couple stress theory. In all these studies, the motion equations have been derived by Hamilton's principle. More recently, non-local EB and TM beam models have been proposed by Pradhan [26] and Yang and Lim [27] based on a stress gradient elasticity theory, the former with a weak formulation of the motion equations in conjunction with finite element analysis [26], and the latter using Hamilton's principle [27]. Specifically, Yang and Lim [27] have used a strain energy density built on a non-local normal stress expressed in terms of higher-order derivatives of the normal strain and, as a result, the corresponding motion equations and boundary conditions (B.C.) involve higher-order derivatives of the non-local bending moment (in this respect, see also Ref. [28, 29]). Yang and Lim [27] have shown that if the motion equations and the pertinent B.C. are not derived by a consistent variational framework and in particular by Hamilton's principle, but instead by a direct replacement of the non-local stress resultants (non-local bending moment and non-local shear) into the classical beam motion equations, some inconsis- 
tencies do arise in terms of equilibrium and B.C. For this reason, the softening effects that those theories predict in the bending stiffness or the natural frequencies cannot be considered, according to Yang and Lim [27], as reliable results. These conclusions have been drawn for both EB and TM beam models.

Along with the studies above, where in general static and dynamic responses of non-local beam models have been investigated, some studies focusing on the static response only are worth mentioning. For instance, Eringen's integral theory has been used by Peddieson et al. [30], Wang and Shindo [31], Civalek and Demir [32] to derive non-local EB beam models, by Wang and Liew [33], Wang and et al. [34] to derive non-local TM beam models, the latter in conjunction with the principle of virtual work. A non-local EB beam model has been presented by Challamel and Wang [35] based on a gradient elastic model and a non-local integral elastic model, where the constitutive relation is expressed by combining local and nonlocal curvatures. A non-local EB model has been also presented by McFarland and Colton [13] based on a micropolar elasticity constitutive law. Lam et al. [12] have investigated a non-local EB model as derived from a general strain gradient elasticity theory, while Park and Gao [36] have studied a non-local EB model based on a modified couple stress theory. The static response of EB beam models based on strain gradient and couple stress theories has been investigated by Chen and Feng [37], Akgöz and Civalek [38].

The purpose of this paper is to illustrate a new nonlocal TM beam model, derived within the framework of a mechanically-based non-local elasticity theory recently proposed by the authors [39-46], where nonlocal effects are modelled as long-range interactions resulting from the relative displacement between volume elements. Consistently with typical engineering beam theories, where the equilibrium of a beam segment is set in an average (weak) sense based on the stress resultants acting on the cross section, in the proposed non-local beam model the long-range interactions are modelled as volume forces and moments, mutually exerted by non-adjacent beam segments, that contribute to the equilibrium of any beam segment along with the local stress resultants. The long-range volume forces/moments are built as linearly depending, through pertinent attenuation functions governing the spatial decay of the non-local effects, on the product of the volumes of the interacting beam segments and on generalized measures of their relative motion. These generalized measures are based on the pure beam deformation modes derived by Fuchs [47, 48], i.e. a "pure axial" symmetric mode, a "pure bending" symmetric mode and a "pure shear" asymmetric mode.

These are the key issues addressed in the paper: (i) the non-local beam model will be cast within a consistent variational framework (on the importance of a consistent variational formulation see, e.g., Ref. [27-29]); in particular, the equilibrium equations will be derived based on a pertinent total elastic potential energy, where local and non-local contributions reflect the mechanical interpretation given to the long-range interactions; (ii) the behavior of the non-local beam model will be investigated for a variety of non-local and geometrical parameters; in this context, a comparison with some alternative models in the literature will be presented; (iii) it will be shown that the nonlocal beam model captures very well experimental data on the static response of cantilever micro-beams [12].

The paper is organized as follows. Upon recalling the basic equations of the local TM beam theory in Sect. 2, the long-range volume forces/moments are introduced in Sect. 3. The equilibrium equations of the non-local TM beam model along with the mechanical B.C. are derived in Sect. 4 by a variational formulation. Numerical results including comparisons with experimental data are presented in Sect. 5.

\section{Local Timoshenko beam theory: basic equations}

In this Section, for the sake of clarity as well as to introduce some basic notations, the classical TM beam theory is briefly summarized.

Consider the initially straight beam of length $L$ and uniform cross section shown in Fig. 1. The material is assumed to be isotropic and linearly elastic. The beam is referred to a Cartesian (orthogonal) coordinate system $O x y z$, where the $x$-axis coincides with the centroidal axis, the $y$-and $z$-axes are principal axes of the cross section, and $x z$ is the bending plane. 
Fig. 1 Shear deformable beam of arbitrary cross section. Positive sign conventions are reported

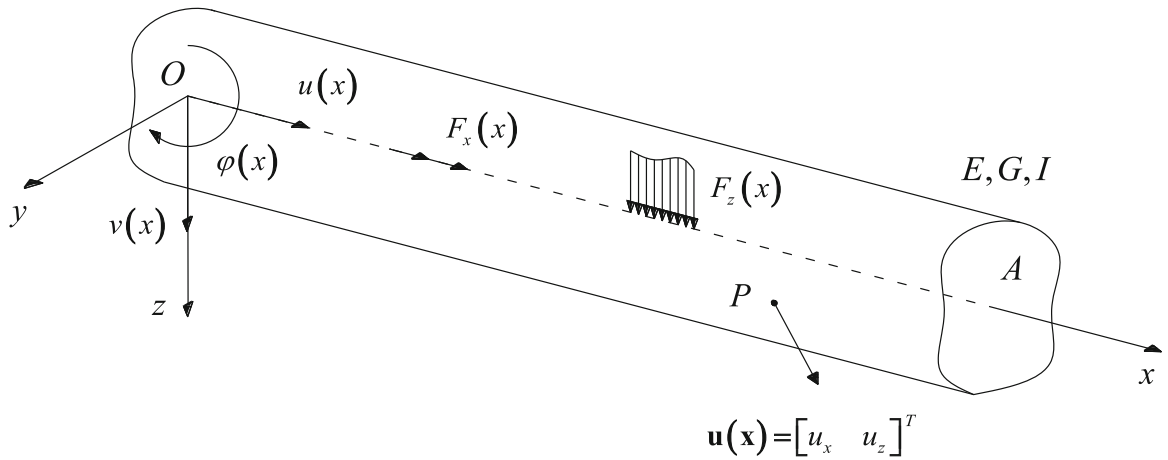

Based on the classical TM beam theory, the displacement field is described as follows:

$u_{x}(\mathbf{x})=u(x)-z \varphi(x) ; u_{y}(\mathbf{x})=0 ; u_{z}(\mathbf{x})=v(x)$,

where $\mathbf{x}=\left[\begin{array}{lll}x & y & z\end{array}\right]^{\mathrm{T}}$ is the position vector; $u(x)$ and $v(x)$ denote the $x$ - and $z$-components of the displacement of a point at $x$ on the centroidal axis and $\varphi(x)$ is the rotation about the $y$-axis. The latter is taken as positive if clockwise. The axial, transverse shear and bending strains ensuing from the above kinematic model are given, respectively, by:

$$
\begin{aligned}
& \varepsilon(x)=\frac{\mathrm{d} u(x)}{\mathrm{d} x} ; \quad \gamma(x)=\frac{\mathrm{d} v(x)}{\mathrm{d} x}-\varphi(x) ; \\
& \chi(x)=-\frac{\mathrm{d} \varphi(x)}{\mathrm{d} x} .
\end{aligned}
$$

The associated stress resultants are the classical local normal stress, shear stress and bending moment, given by:

$$
\begin{aligned}
& N^{(l)}(x)=\int_{A} \sigma_{x}^{(l)}(\mathbf{x}) \mathrm{d} A ; \quad T^{(l)}(x)=\int_{A} \tau_{x z}^{(l)}(\mathbf{x}) \mathrm{d} A ; \\
& M^{(l)}(x)=\int_{A} \sigma_{x}^{(l)}(\mathbf{x}) z \mathrm{~d} A
\end{aligned}
$$

where the superscript in parentheses means local, while $\sigma_{x}^{(l)}(\mathbf{x})$ and $\tau_{x z}^{(l)}(\mathbf{x})$ are two of the six components of the Cauchy stress tensor gathered into the vector $\boldsymbol{\sigma}^{(l)}(\mathbf{x})=\left[\begin{array}{llllll}\sigma_{x}^{(l)} & \sigma_{y}^{(l)} & \sigma_{z}^{(l)} & \tau_{y z}^{(l)} & \tau_{x z}^{(l)} & \tau_{x y}^{(l)}\end{array}\right]^{\mathrm{T}}$.

Collecting the stress resultants $(3 \mathrm{a}-\mathrm{c})$ and generalized strain components $(2 \mathrm{a}-\mathrm{c})$ into the vectors $\mathbf{s}^{(l)}$ $(x)=\left[\begin{array}{lll}N^{(l)}(x) & T^{(l)}(x) & M^{(l)}(x)\end{array}\right]^{\mathrm{T}} \quad$ and $\quad \mathbf{d}^{(l)}(x)=$ $\left[\begin{array}{lll}\varepsilon(x) & \gamma(x) \chi(x)\end{array}\right]^{\mathrm{T}}$, the linear-elastic constitutive laws can be written in compact form as:

$\mathbf{s}^{(l)}(x)=\mathbf{D}^{*} \mathbf{d}^{(l)}(x)$

where $\mathbf{D}^{*}=\operatorname{Diag}\left[\begin{array}{lll}E^{*} A & K_{s} G^{*} A & E^{*} I\end{array}\right]$, with $E^{*}=$ $\beta_{1} E$ and $G^{*}=\beta_{1} G, E$ and $G$ being Young's modulus and shear modulus respectively, whereas $0 \leq \beta_{1} \leq 1$ is a dimensionless real coefficient weighting the amount of local effects when the beam model includes also long-range interactions [42-44], as will be outlined in the next Section; $A$ and $I$ are the area and moment of inertia of the cross section; $K_{S}$ is the shear correction factor. Obviously, the classical local TM beam parameters correspond to $\beta_{1}=1$, so that $E^{*}=E$ and $G^{*}=G$.

Denoting by $F_{x}(x)$ and $F_{z}(x)$ the external forces per unit length in the $x$ - and $z$-directions, the equilibrium of the TM beam is governed by the following equations:

$$
\begin{aligned}
& \frac{\mathrm{d} N^{(l)}(x)}{\mathrm{d} x}+F_{x}(x)=0 ; \\
& \frac{\mathrm{d} T^{(l)}(x)}{\mathrm{d} x}+F_{z}(x)=0 ; \\
& \frac{\mathrm{d} M^{(l)}(x)}{\mathrm{d} x}-T^{(l)}(x)=0 .
\end{aligned}
$$

The pertinent kinematic B.C. read:

$$
\begin{aligned}
u\left(x_{i}\right)=u_{i} ; \quad v\left(x_{i}\right)=v_{i} ; \quad \varphi\left(x_{i}\right)=\varphi_{i} ; \quad i= & 0, L \\
& (6 \mathrm{a}-\mathrm{c})
\end{aligned}
$$

being $u_{i}, v_{i}$ and $\varphi_{i}$ the displacements and rotations at the beam ends, i.e. at $x_{0}=0$ and $x_{L}=L$. Finally, if $N_{i}$, $M_{i}$ and $T_{i}$ denote the external forces/moments acting at the ends of the beam $(i=0, L)$, the mechanical B.C. are given by: 
$N^{(l)}\left(x_{i}\right)=\mp N_{i} ; \quad T^{(l)}\left(x_{i}\right)=\mp T_{i} ; \quad M^{(l)}\left(x_{i}\right)= \pm M_{i}$.

$(7 \mathrm{a}-\mathrm{c})$

\section{Non-local Timoshenko beam model with long- range interactions}

This Section presents the main features of the proposed non-local beam model. It is derived within the framework of a mechanically-based approach to non-local elasticity theory [39-46], where non-local effects are represented as long-range interactions exchanged by non-adjacent volume elements as a result of their relative motion. Herein, such a general idea is applied within the context of typical engineering beam theories, where the equilibrium of a beam segment is set in an average (weak) sense based on the stress resultants acting on the cross section. In particular, the long-range interactions are modeled as volume forces and moments, exchanged by nonadjacent beam segments as a result of their relative motion measured by the pure deformation modes of the beam [47, 48]. As will be outlined next, this modeling of long-range interactions can be interpreted on a meaningful mechanical basis, as corresponds to assuming a spring-like connection between couples of non-adjacent beam segments where pure axial, pure bending and pure shear long-range springs (with distance-decaying stiffness) can be separately accounted for. In the following, the analytical expressions and mechanical interpretation of the long-range volume forces/moments are given. Hence, the equilibrium equations are built by direct introduction of the long-range volume forces/moments in the standard equilibrium equations of a beam segment. A similar approach allows to derive the mechanical B.C.

\subsection{Long-range resultants}

By means of an eigenvalue analysis, Fuchs [47, 48] decomposed a TM beam element into three unimodal components acting in parallel: a "pure axial", a "pure bending" and a "pure shear" element. Such decomposition yields uncoupled constitutive laws between generalized stress and strain measures associated with each deformation mode. Fuchs [47, 48] provided a clear geometrical interpretation of the deformation modes: the "pure axial" mode is a symmetric mode defined by the relative axial displacements between the ends; the "pure bending" mode is a symmetric mode defined by the relative rotation between the ends; the "pure shear" mode is an asymmetric mode defined by the relative rotation between the ends, with respect to the line given by the relative transverse displacements. Furthermore, Fuchs [47, 48] derived the self-equilibrated forces/moments at the ends of the TM beam element resulting from the pure deformation modes.

In order to define the long-range interactions based on Fuchs' unimodal components, a discrete model of the beam is built by dividing the beam domain in $N$ segments of length $\Delta x$, so that $x_{i}=i \Delta x$ with $i=0,1, \ldots, N-1\left(x_{N}=x_{L}\right)$, defines the position of the segment $\Delta V\left(x_{i}\right)=A \Delta x$ along the axis (see Fig. 2).

It is assumed that the equilibrium of each beam segment is attained due to: (i) the local stress resultants in Eq. (3) exerted by the adjacent beam segments; (ii) the resultants of the volume forces/moments, $R_{x}, R_{z}$ and $R_{\varphi}$ exerted by all the non-adjacent beam segments. Such resultants are shown in Fig. 2 where the equilibrium of an elementary beam segment located at $x=x_{i}$ is displayed.

The key assumption of the proposed non-local beam model is that the long-range volume forces/moments mutually exerted by two non-adjacent beam segments $\Delta V\left(x_{i}\right)$ and $\Delta V\left(\xi_{k}\right)$ located, respectively, at $x=x_{i}$ and $x=\xi_{k}$, arise due to their relative motion described by Fuchs' generalized measures of relative displacement/rotations (see Fig. 3) i.e., the relative axial displacement:

$\eta\left(x_{i}, \xi_{k}\right)=u\left(\xi_{k}\right)-u\left(x_{i}\right) ;$

the relative rotation:

$\theta\left(x_{i}, \xi_{k}\right)=\varphi\left(\xi_{k}\right)-\varphi\left(x_{i}\right) ;$

and the rotation with respect to the line given by the relative transverse displacement:

$$
\begin{aligned}
\psi\left(x_{i}, \xi_{k}\right)= & {\left[\frac{v\left(\xi_{k}\right)-v\left(x_{i}\right)}{\xi_{k}-x_{i}}-\varphi\left(\xi_{k}\right)\right] } \\
& +\left[\frac{v\left(\xi_{k}\right)-v\left(x_{i}\right)}{\xi_{k}-x_{i}}-\varphi\left(x_{i}\right)\right] .
\end{aligned}
$$

Based on the analytical expressions of the generalized stress measures derived by Fuchs [47, 48] for each unimodal component, the long-range volume forces/moments are taken as proportional to the pure 
Fig. 2 Equilibrium of an elementary beam segment: a axial; b transverse; c bending

(a)

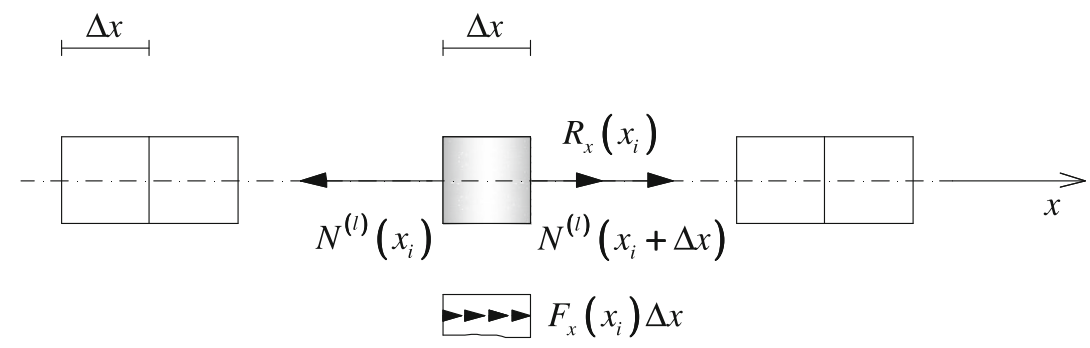

(b)

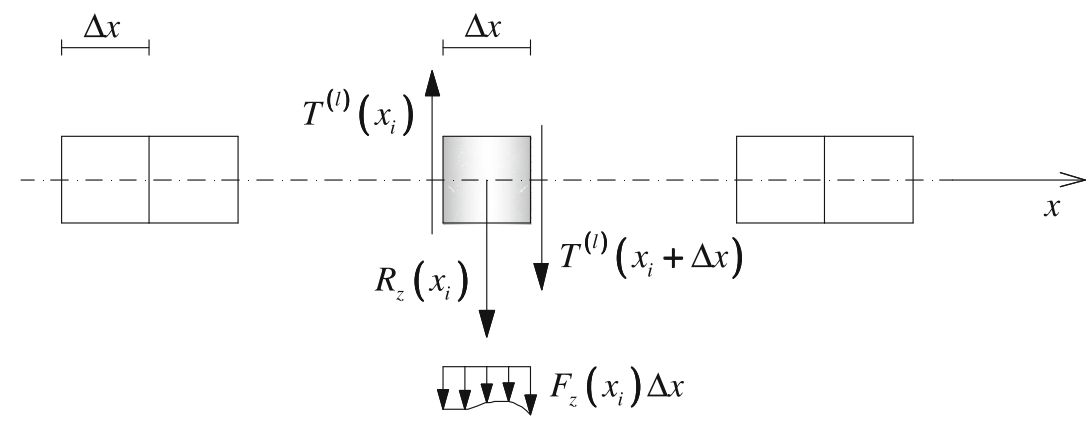

(c)

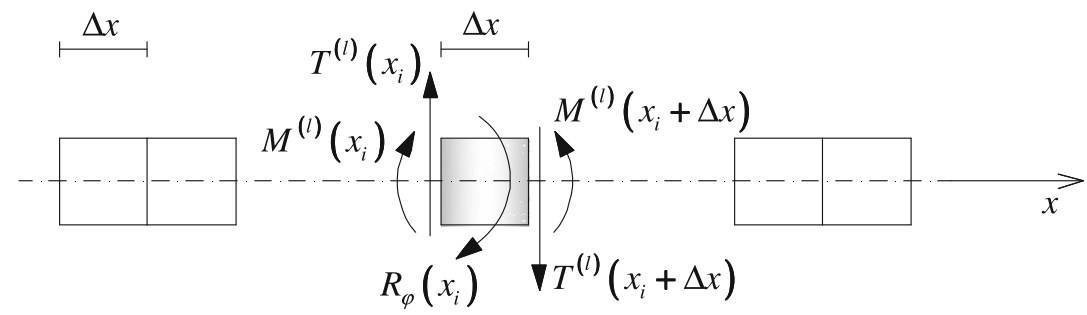

deformation modes. By analogy to the mechanicallybased model of non-local bar proposed by Di Paola et al. [42, 45, 46], a linear dependence on the product of the volumes of the interacting beam segments through appropriate attenuation functions governing the spatial decay of non-local effects is also included.

The pure axial deformation mode (see Fig. 3a) gives rise to long-range volume axial forces $r_{x}\left(x_{i}, \xi_{k}\right)$ mutually exerted by two non-adjacent beam segments $\Delta V\left(x_{i}\right)$ and $\Delta V\left(\xi_{k}\right)$, as a result of their relative axial displacement $\eta\left(x_{i}, \xi_{k}\right)$, i.e.:

$r_{x}\left(x_{i}, \xi_{k}\right)=q_{x}\left(x_{i}, \xi_{k}\right) \Delta V\left(x_{i}\right) \Delta V\left(\xi_{k}\right) ;$

$q_{x}\left(x_{i}, \xi_{k}\right)=g_{x}\left(x_{i}, \xi_{k}\right) \eta\left(x_{i}, \xi_{k}\right)$

where $q_{x}\left(x_{i}, \xi_{k}\right)$ given by Eq. (11b) is the specific long-range axial force depending on the relative axial displacement (8), through an appropriate symmetric real-valued attenuation function $g_{x}(x, \xi)$ governing the spatial decay of non-local axial effects.
The pure bending mode (see Fig. 3b) gives rise to long-range volume moments $r_{\varphi \varphi}\left(x_{i}, \xi_{k}\right)$ mutually exerted by two non-adjacent beam segments $\Delta V\left(x_{i}\right)$ and $\Delta V\left(\xi_{k}\right)$ as a result of their relative rotation $\theta\left(x_{i}, \xi_{k}\right)$, i.e.:

$$
\begin{aligned}
& r_{\varphi \varphi}\left(x_{i}, \xi_{k}\right)=q_{\varphi \varphi}\left(x_{i}, \xi_{k}\right) \Delta V\left(x_{i}\right) \Delta V\left(\xi_{k}\right) \\
& q_{\varphi \varphi}\left(x_{i}, \xi_{k}\right)=g_{\varphi}\left(x_{i}, \xi_{k}\right) \theta\left(x_{i}, \xi_{k}\right)
\end{aligned}
$$

where $q_{\varphi \varphi}\left(x_{i}, \xi_{k}\right)$ given by Eq. (12b) is the specific long-range moment proportional to the relative rotation (9) through an appropriate symmetric realvalued distance-decaying attenuation function $g_{\varphi}\left(x_{i}, \xi_{k}\right)$.

Finally, the pure shear mode (see Fig. 3c) gives rise to long-range volume transverse forces and moments, $r_{z}\left(x_{i}, \xi_{k}\right)$ and $r_{\varphi z}\left(x_{i}, \xi_{k}\right)$, mutually exerted by two nonadjacent beam segments $\Delta V\left(x_{i}\right)$ and $\Delta V\left(\xi_{k}\right)$ as a result 
Fig. 3 Pure deformation modes of the Timoshenko beam and associated longrange springs: $\mathbf{a}$ axial; $\mathbf{b}$ pure bending; c pure shear (a)

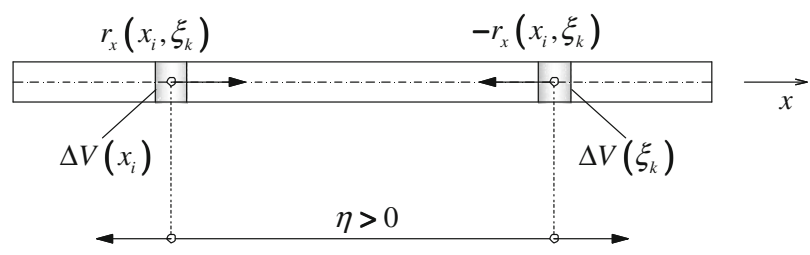

(b)

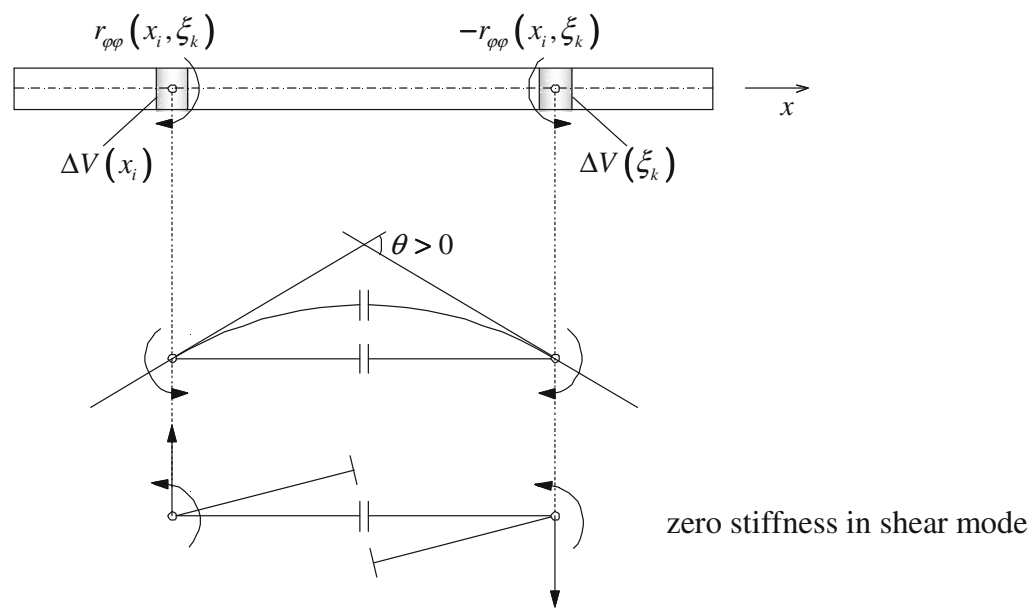

(c)

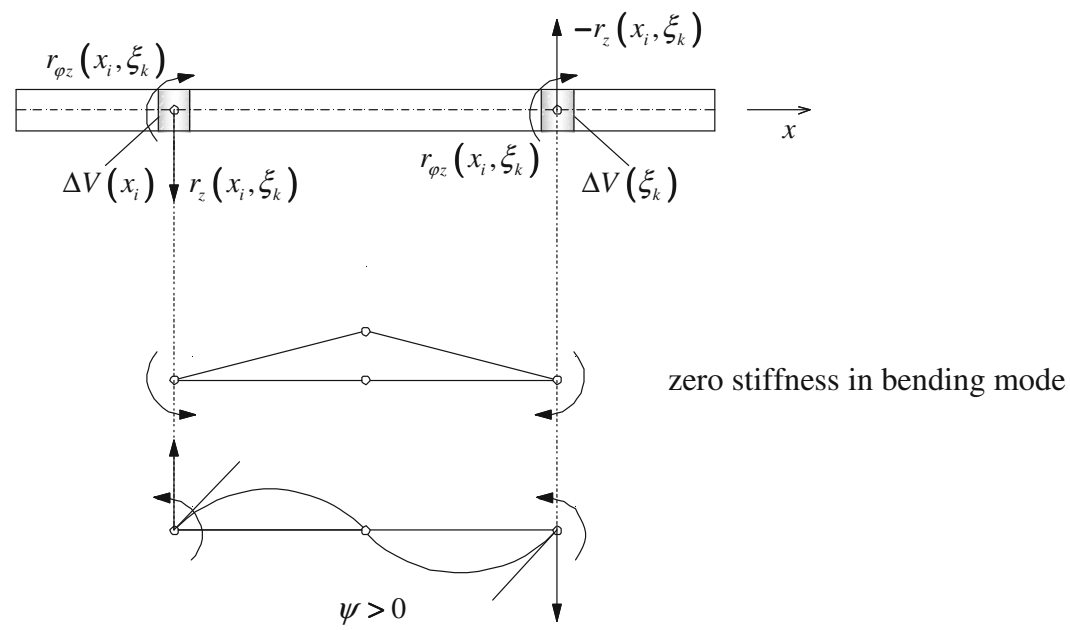

of their rotations with respect to the line given by the relative transverse displacement, $\psi\left(x_{i}, \xi_{k}\right)$, i.e.:

$r_{z}\left(x_{i}, \xi_{k}\right)=q_{z}\left(x_{i}, \xi_{k}\right) \Delta V\left(x_{i}\right) \Delta V\left(\xi_{k}\right) ;$

$q_{z}\left(x_{i}, \xi_{k}\right)=2 \frac{\operatorname{sgn}\left(\xi_{k}-x_{i}\right)}{\left|\xi_{k}-x_{i}\right|} g_{z}\left(x_{i}, \xi_{k}\right) \psi\left(x_{i}, \xi_{k}\right) \quad(13 \mathrm{a}-\mathrm{b})$

$$
\begin{aligned}
& r_{\varphi z}\left(x_{i}, \xi_{k}\right)=q_{\varphi z}\left(x_{i}, \xi_{k}\right) \Delta V\left(x_{i}\right) \Delta V\left(\xi_{k}\right) ; \\
& q_{\varphi z}\left(x_{i}, \xi_{k}\right)=g_{z}\left(x_{i}, \xi_{k}\right) \psi\left(x_{i}, \xi_{k}\right)
\end{aligned}
$$

In the previous equations, $q_{z}\left(x_{i}, \xi_{k}\right)$ in Eq. (13b) and $q_{\varphi z}\left(x_{i}, \xi_{k}\right)$ in Eq. (14b) are the specific long-range volume transverse forces and moments, depending on $\psi\left(x_{i}, \xi_{k}\right)$ through a symmetric real-valued distance- 
decaying attenuation function $g_{z}\left(x_{i}, \xi_{k}\right)$. In Eq. (13b), $\operatorname{sgn}(\xi-x)=+1$ if $(\xi-x)>0$ and $\operatorname{sgn}(\xi-x)=-1$ if $(\xi-x)<0$ is introduced to ensure consistency of $r_{z}(x, \xi)$ with the sign convention of the long-range resultants in Fig. 2. Hence, the total long-range volume moments due to bending and shear deformation modes can be expressed as:

$$
\begin{aligned}
& r_{\varphi}\left(x_{i}, \xi_{k}\right)=q_{\varphi}\left(x_{i}, \xi_{k}\right) \Delta V\left(x_{i}\right) \Delta V\left(\xi_{k}\right) ; \\
& q_{\varphi}\left(x_{i}, \xi_{k}\right)=q_{\varphi \varphi}\left(x_{i}, \xi_{k}\right)+q_{\varphi z}\left(x_{i}, \xi_{k}\right) .
\end{aligned}
$$

Based on the above definitions, the resultants of the long-range volume forces/moments exerted on the beam segment $\Delta V\left(x_{i}\right)$ at $x=x_{i}$ by all the non-adjacent beam segments $\Delta V\left(\xi_{k}\right)$ at $x=\xi_{k}, \xi_{k} \neq x_{i}$, can be obtained as follows:

$$
\begin{aligned}
R_{x}\left(x_{i}\right) & =\sum_{k=0, k \neq i}^{N-1} r_{x}\left(x_{i}, \xi_{k}\right) ; \\
R_{z}\left(x_{i}\right) & =\sum_{k=0, k \neq i}^{N-1} r_{z}\left(x_{i}, \xi_{k}\right) ; \\
R_{\varphi}\left(x_{i}\right) & =\sum_{k=0, k \neq i}^{N-1} r_{\varphi}\left(x_{i}, \xi_{k}\right) .
\end{aligned}
$$

For brevity, $R_{x}\left(x_{i}\right), R_{z}\left(x_{i}\right)$ and $R_{\varphi}\left(x_{i}\right)$ hereinafter will be referred to as long-range resultants.

A close inspection of the above equations suggests that the long-range volume forces/moments can be interpreted as the result of three spring-like connections between non-adjacent beam segments which separately account for pure axial, pure bending and pure shear modes (see Fig. 3). Thus, from a mechanical point of view, the proposed non-local beam model is conceptually equivalent to the non-local bar built by Di Paola et al. [42, 45, 46], where the longrange volume axial forces are represented, within the context of a discrete model, as linearly-elastic springs of distance-decaying stiffness connecting non-adjacent volume elements. In this regard it worth remarking that, if the long-range volume transverse forces/moments were taken as depending on the relative transverse displacement and not on the pure shear deformation (10), long-range volume transverse forces/moments would erroneously arise from a relative transverse displacement induced, for instance, by a rigid rotation of the beam. In this sense, it can be stated that the proposed non-local beam model is invariant with respect to rigid body motion and that the axial, bending and shear non-local behaviors are mechanically consistent.

Finally, some remarks are in order on the choice of the attenuation functions $g_{s}(x, \xi)$, with $s=x, z, \varphi$, governing the spatial decay of non-local effects. To ensure that the long-range resultants have a restoring nature, for any couple of interacting beam segments, the attenuation functions must be strictly positive. Furthermore, these functions are taken as symmetric functions with respect to the arguments $x$ and $\xi$, to ensure that the long-range resultants exchanged by the interacting beam segments are mutual, according to Newton's third law.

To make the beam model as general and versatile as possible, three different functions $g_{s}(x, \xi)$, with $s=x, z, \varphi$, have been introduced in the definition of the long-range forces/moments. Indeed, non-local axial, bending or shear effects may exhibit a different spatial decay depending on the material microstructure. In general, both the mathematical form of the attenuation functions [39-46, 49, 51] and the pertinent parameters depend on the material and should be determined based on experimental evidence.

\subsection{Equilibrium equations}

Within the context of the proposed non-local beam model, each beam segment is in equilibrium under: (i) the local stress resultants $(3 a-c)$ exerted by the adjacent beam segments; (ii) the resultants of the volume forces/moments exerted by all non-adjacent beam segments [see Eqs. (16a-c)]; and the external forces represented here by the unit length forces $F_{x}(x)$ and $F_{z}(x)$. Then, the equilibrium equations of the beam segment $\Delta V\left(x_{i}\right)=A \Delta x$ at $x=x_{i}$, for $i=0,1, \ldots$, $N-1$, read (see Fig. 2):

$$
\begin{aligned}
& N^{(l)}\left(x_{i}+\Delta x\right)-N^{(l)}\left(x_{i}\right)+R_{x}\left(x_{i}\right)+F_{x}\left(x_{i}\right) \Delta x=0 ; \\
& T^{(l)}\left(x_{i}+\Delta x\right)-T^{(l)}\left(x_{i}\right)+R_{z}\left(x_{i}\right)+F_{z}\left(x_{i}\right) \Delta x=0 ; \\
& M^{(l)}\left(x_{i}+\Delta x\right)-M^{(l)}\left(x_{i}\right)-T^{(l)}\left(x_{i}\right) \Delta x-R_{\varphi}\left(x_{i}\right)=0 .
\end{aligned}
$$

Substituting Eqs. (16a-c) for the long-range resultants $R_{x}\left(x_{i}\right), R_{z}\left(x_{i}\right)$ and $R_{\varphi}\left(x_{i}\right)$ into Eqs. (17a-c), dividing both sides by $\Delta x$ and taking the limit as $\Delta x \rightarrow 0$, the following integro-differential equilibrium equations are obtained: 


$$
\begin{aligned}
& \frac{\mathrm{d} N^{(l)}(x)}{\mathrm{d} x}+A^{2} \int_{0}^{L} q_{x}(x, \xi) \mathrm{d} \xi+F_{x}(x)=0 ; \\
& \frac{\mathrm{d} T^{(l)}(x)}{\mathrm{d} x}+A^{2} \int_{0}^{L} q_{z}(x, \xi) \mathrm{d} \xi+F_{z}(x)=0 ; \\
& \frac{\mathrm{d} M^{(l)}(x)}{\mathrm{d} x}-T^{(l)}(x)-A^{2} \int_{0}^{L} q_{\varphi}(x, \xi) \mathrm{d} \xi=0 .
\end{aligned}
$$

Notice that Eqs. (18a-c) differ from the differential equilibrium equations of the local TM beam $(5 \mathrm{a}-\mathrm{c})$ just for the integral terms representing the long-range resultants per unit length.

The B.C. associated with Eqs. $(18 \mathrm{a}-\mathrm{c})$ coincide with the ones pertaining to the local beam, given in Eqs. (6) and (7). Indeed, at the beam ends the longrange resultants can be considered as infinitesimal of higher order with respect to the local stress resultants (see Ref. [42-44]).

\section{Variational formulation}

In this Section, the equilibrium equations of the nonlocal TM beam along with the pertinent B.C. are derived within a variational framework by applying the minimum potential energy principle.

A variational formulation of the equations governing the proposed non-local beam model can be pursued starting from the work identity [42-44]:

$$
\begin{aligned}
& \int_{0}^{L} \mathbf{s}^{(l) \mathrm{T}}(x) \mathbf{d}^{(l)}(x) \mathrm{d} x \\
& =\int_{0}^{L} \mathbf{F}^{\mathrm{T}}(x) \mathbf{u}(x) \mathrm{d} x+\mathbf{s}_{0}^{\mathrm{T}} \mathbf{u}_{0}+\mathbf{s}_{L}^{\mathrm{T}} \mathbf{u}_{L} \\
& +A^{2} \int_{0}^{L} \int_{0}^{L} \tilde{\mathbf{q}}^{\mathrm{T}}(x, \xi) \mathbf{u}(x) \mathrm{d} x \mathrm{~d} \xi
\end{aligned}
$$

where $\mathbf{s}^{(l)}(x)$ and $\mathbf{d}^{(l)}(x)$ are the vectors collecting the local stress resultants $(3 \mathrm{a}-\mathrm{c})$ and generalized strain components $(2 \mathrm{a}-\mathrm{c})$, respectively, defined in Sect. 2; while the vectors $\mathbf{u}(x), \mathbf{F}(x)$ and $\tilde{\mathbf{q}}(x, \xi)$ are given by:

$$
\begin{aligned}
& \mathbf{u}(x)=\left[\begin{array}{lll}
u(x) & v(x) & \varphi(x)
\end{array}\right]^{\mathrm{T}} \\
& \mathbf{F}(x)=\left[\begin{array}{lll}
F_{x}(x) & F_{z}(x) & 0
\end{array}\right]^{\mathrm{T}} ; \\
& \tilde{\mathbf{q}}(x, \xi)=\left[\begin{array}{lll}
q_{x}(x, \xi) & q_{z}(x, \xi) & q_{\varphi}(x, \xi)
\end{array}\right]^{\mathrm{T}} .
\end{aligned}
$$$$
(20 a-c)
$$

Furthermore, in Eq. (19), $\mathbf{s}_{i}(i=0, L)$ is the vector listing the axial force, transverse force and moment at the beam ends, $N_{i}, T_{i}$ and $M_{i}, \quad(i=0, L)$; the corresponding displacements and rotations are gathered into vector $\mathbf{u}_{i}(i=0, L)$.

The double integral on the r.h.s of Eq. (19) is obtained as the continuous counterpart of the work done by the long-range resultants $(16 \mathrm{a}-\mathrm{c})$ exchanged between non-adjacent beam segments of the discrete model built in the previous Section (see Fig. 2), i.e.:

$$
\begin{aligned}
& \lim _{\Delta x, \Delta \xi \rightarrow 0} \sum_{i=0}^{N-1} \mathbf{R}^{\mathrm{T}}\left(x_{i}\right) \mathbf{u}\left(x_{i}\right) \\
& =\lim _{\Delta x, \Delta \xi \rightarrow 0} A^{2} \sum_{i=0}^{N-1} \sum_{k=0, k \neq i}^{N-1} \tilde{\mathbf{q}}^{\mathrm{T}}\left(x_{i}, \xi_{k}\right) \mathbf{u}\left(x_{i}\right) \Delta x \Delta \xi \\
& =A^{2} \int_{0}^{L} \int_{0}^{L} \tilde{\mathbf{q}}^{\mathrm{T}}(x, \xi) \mathbf{u}(x) \mathrm{d} x \mathrm{~d} \xi
\end{aligned}
$$

where $\mathbf{R}\left(x_{i}\right)=\left[\begin{array}{lll}R_{x}\left(x_{i}\right) & R_{z}\left(x_{i}\right) & R_{\varphi}\left(x_{i}\right)\end{array}\right]^{\mathrm{T}}$.

Due to the symmetry of the attenuation functions $g_{s}(x, \xi), s=x, z, \varphi$, with respect to the arguments $x$ and $\xi$, the following identity holds (see Appendix 1):

$$
\begin{aligned}
& \int_{0}^{L} \int_{0}^{L} \tilde{\mathbf{q}}^{\mathrm{T}}(x, \xi) \mathbf{u}(x) \mathrm{d} x \mathrm{~d} \xi \\
& =-\frac{1}{2} \int_{0}^{L} \int_{0}^{L} \mathbf{q}^{\mathrm{T}}(x, \xi) \mathbf{e}(x, \xi) \mathrm{d} x \mathrm{~d} \xi
\end{aligned}
$$

where

$$
\begin{aligned}
& \mathbf{e}(x, \xi)=\left[\begin{array}{lll}
\eta(x, \xi) & \theta(x, \xi) & \psi(x, \xi)
\end{array}\right]^{\mathrm{T}} \\
& \mathbf{q}(x, \xi)=\left[\begin{array}{lll}
q_{x}(x) & q_{\varphi \varphi}(x, \xi) & q_{\varphi z}(x, \xi)
\end{array}\right]^{\mathrm{T}}
\end{aligned}
$$

are the vectors collecting the generalized measures of relative motion and the associated specific-long-range forces/moments, respectively. Vectors $\mathbf{q}(x, \xi)$ and $\mathbf{e}(x, \xi)$ are related by Eq. (11b), Eq. (12b), and Eq. (14b), which can be rewritten in compact form as:

$\mathbf{q}(x, \xi)=\mathbf{G}(x, \xi) \mathbf{e}(x, \xi)$

where

$$
\mathbf{G}(x, \xi)=\operatorname{Diag}\left[g_{x}(x, \xi) \quad g_{\varphi}(x, \xi) \quad g_{z}(x, \xi)\right]
$$

is a diagonal matrix listing the attenuation functions. Equation (24) may be viewed as the constitutive law between the specific long-range forces and the associated generalized measures of relative motion.

Taking into account Eq. (22), the work identity can be rewritten as follows: 


$$
\begin{gathered}
\int_{0}^{L} \mathbf{s}^{(l) \mathrm{T}}(x) \mathbf{d}^{(l)}(x) \mathrm{d} x+\frac{A^{2}}{2} \int_{0}^{L} \int_{0}^{L} \mathbf{q}^{\mathrm{T}}(x, \xi) \mathbf{e}(x, \xi) \mathrm{d} x \mathrm{~d} \xi \\
=\int_{0}^{L} \mathbf{F}^{\mathrm{T}}(x) \mathbf{u}(x) \mathrm{d} x+\mathbf{s}_{0}^{\mathrm{T}} \mathbf{u}_{0}+\mathbf{s}_{L}^{\mathrm{T}} \mathbf{u}_{L} .
\end{gathered}
$$

Based on Eq. (26), the elastic potential energy stored in the TM beam with long-range interactions can be defined as sum of local and non-local contributions:

$$
\begin{aligned}
& \Phi\left(\mathbf{d}^{(l)}(x), \mathbf{e}(x, \xi)\right) \\
& =\int_{0}^{L} \phi^{(l)}\left(\mathbf{d}^{(l)}(x)\right) \mathrm{d} x+\frac{A^{2}}{2} \int_{0}^{L} \int_{0}^{L} \phi^{(n l)}(\mathbf{e}(x, \xi)) \mathrm{d} x \mathrm{~d} \xi \\
& =\frac{1}{2} \int_{0}^{L} \mathbf{d}^{(l) \mathrm{T}}(x) \mathbf{D}^{*} \mathbf{d}^{(l)}(x) \mathrm{d} x \\
& +\frac{A^{2}}{4} \int_{0}^{L} \int_{0}^{L} \mathbf{e}^{\mathrm{T}}(x, \xi) \mathbf{G}(x, \xi) \mathbf{e}(x, \xi) \mathrm{d} x \mathrm{~d} \xi
\end{aligned}
$$

where the linear-elastic constitutive laws (4) and Eq. (24) have been introduced. In the previous equation, $\phi^{(l)}\left(\mathbf{d}^{(l)}(x)\right)$ and $\phi^{(n l)}(\mathbf{e}(x, \xi))$ denote the local and non-local elastic potential energy per unit length, respectively. The consistency of the elastic potential energy (27) may be assessed by deriving $\phi^{(l)}\left(\mathbf{d}^{(l)}(x)\right)$ and $\phi^{(n l)}(\mathbf{e}(x, \xi))$ with respect to the pertinent state variables, i.e.:

$$
\begin{aligned}
\mathbf{s}^{(l)}(x) & =\frac{\partial \phi^{(l)}}{\partial \mathbf{d}^{(l)}}=\mathbf{D}^{*} \mathbf{d}^{(l)}(x) ; \\
\mathbf{q}(x, \xi) & =\frac{\partial \phi^{(n l)}}{\partial \mathbf{e}}=\mathbf{G}(x, \xi) \mathbf{e}(x, \xi) .
\end{aligned}
$$

These relationships are coincident with the constitutive equations for the local stress resultants (4) and the specific long-range forces/moments (24).

Taking into account Eq. (22), the first variation of the elastic potential energy (27) can be written as:

$$
\begin{aligned}
\delta \Phi\left(\mathbf{d}^{(l)}(x), \mathbf{u}(x)\right)= & \int_{0}^{L} \mathbf{d}^{(l) \mathrm{T}}(x) \mathbf{D}^{*} \delta \mathbf{d}^{(l)}(x) \mathrm{d} x \\
& -A^{2} \int_{0}^{L} \int_{0}^{L} \tilde{\mathbf{q}}(x, \xi) \delta \mathbf{u}(x) \mathrm{d} x \mathrm{~d} \xi .
\end{aligned}
$$

The equilibrium equations and the associated natural B.C. of the non-local TM beam can be derived by enforcing that the first variation of the total potential energy functional $\Pi=\Phi-W_{\text {ext }}$ vanishes in correspondence of the solution, i.e.:

$$
\begin{aligned}
\delta \Pi\left(\mathbf{d}^{(l)}(x), \mathbf{u}(x)\right)= & \delta \Phi\left(\mathbf{d}^{(l)}(x), \mathbf{u}(x)\right) \\
& -\delta W_{\text {ext }}(\mathbf{u}(x))=0
\end{aligned}
$$

where

$$
\delta W_{e x t}(\mathbf{u}(x))=\int_{0}^{L} \mathbf{F}^{\mathrm{T}}(x) \delta \mathbf{u}(x) \mathrm{d} x+\mathbf{s}_{0}^{\mathrm{T}} \delta \mathbf{u}_{0}+\mathbf{s}_{L}^{\mathrm{T}} \delta \mathbf{u}_{L}
$$

is the first variation of the work done by the external forces.

Taking into account the local strain-displacement relationships ( $2 \mathrm{a}-\mathrm{c})$, and applying the standard rules of variational calculus along with integration by parts, the stationarity condition (30) yields the following Euler-Lagrange equations:

$$
\begin{aligned}
& E^{*} A \frac{\mathrm{d}^{2} u(x)}{\mathrm{d} x^{2}}+A^{2} \int_{0}^{L} q_{x}(x, \xi) \mathrm{d} \xi+F_{x}(x)=0 \\
& K_{s} G^{*} A\left(\frac{\mathrm{d}^{2} v(x)}{\mathrm{d} x^{2}}-\frac{\mathrm{d} \varphi(x)}{\mathrm{d} x}\right) \\
& \quad+A^{2} \int_{0}^{L} q_{z}(x, \xi) \mathrm{d} \xi+F_{z}(x)=0 \\
& E^{*} I \frac{\mathrm{d}^{2} \varphi(x)}{\mathrm{d} x^{2}}+K_{s} G^{*} A\left(\frac{\mathrm{d} v(x)}{\mathrm{d} x}-\varphi(x)\right) \\
& \quad+A^{2} \int_{0}^{L} q_{\varphi}(x, \xi) \mathrm{d} \xi=0 .
\end{aligned}
$$

The associated static and kinematic B.C. read:

$$
\begin{aligned}
& N^{(l)}\left(x_{i}\right)=\left.E^{*} A \frac{\mathrm{d} u(x)}{\mathrm{d} x}\right|_{x=x_{i}}=\mp N_{i} ; \text { or } u\left(x_{i}\right)=u_{i} ; \\
& T^{(l)}\left(x_{i}\right)=\left.K_{s} G^{*} A\left(\frac{\mathrm{d} v(x)}{\mathrm{d} x}-\varphi(x)\right)\right|_{x=x_{i}}=\mp T_{i} ; \\
& \text { or } v\left(x_{i}\right)=v_{i} ; \\
& M^{(l)}\left(x_{i}\right)=-\left.E^{*} I \frac{\mathrm{d} \varphi(x)}{\mathrm{d} x}\right|_{x=x_{i}}= \pm M_{0} ; \text { or } \varphi\left(x_{i}\right) \\
& =\varphi_{i},(i=0, L) .
\end{aligned}
$$

Notice that Eqs. (32) and (33) coincide with the equilibrium equations and B.C. derived in Sect. 3.2 on a mechanical basis, once the local stress resultants 
$N^{(l)}(x), T^{(l)}(x), M^{(l)}(x)$ are expressed in terms of the kinematic variables $u(x), v(x)$ and $\varphi(x)$ by using the strain-displacement and constitutive relationships (2) and (4). This result demonstrates the variational consistency of the proposed non-local beam model. In this regard, it worth remarking that the fully local nature of the B.C. has been derived also on a variational basis.

An important remark concerns the B.C. (33): the fact that they hold the same form of classical local theory is a remarkable advantage, which allows standard solution methods, such as the finite difference method or the finite element method, to be applied for solving the equilibrium Eqs. (32) in a straightforward manner. In particular, notice that the variational formulation above can be used for deriving a finite element formulation of the equilibrium equations, as customary in the mechanics of elastic solids. On further advantages involved by the fact that the B.C. hold the same form of classical local theory, in particular with respect to lattice mechanics models, comments can be found in a previous paper by the authors [42], and are not repeated here for brevity.

\section{Numerical applications}

The applications focus on the flexural response of the proposed non-local beam model. Firstly, theoretical results are presented for epoxy micro-beams of rectangular cross section of width $b$ and thickness $h$. Simplysupported and cantilever beams will be considered as study cases. Secondly, it will be shown that the proposed non-local beam model can capture the experimental static response of a cantilever epoxy micro-beam subjected to a tip load, as reported by Lam et al. [12].

In all cases, it will be assumed that pure bending and shear behaviors are governed by the same attenuation functions, i.e. $g_{s}(x, \xi)=g(x, \xi)$, $s=\varphi, z$, which are given here an exponential form:

$g(x, \xi)=\frac{C}{h^{2}} \exp \left(-\frac{|x-\xi|}{l}\right)$

where $C$ is a constant; $h$ denotes the thickness of the cross section; $l$ is an internal length. The larger is the internal length $l$, the wider is the so-called influence distance, i.e. the maximum distance beyond which the attenuation functions and thus the non-local effects become negligible. Further, in the local constitutive Eq. (4) $\beta_{1}=1$ is selected. As a result of this choice for $\beta_{1}$, the non-local solution will tend to the solution obtained by the classical local TM theory, as $l \rightarrow 0$ in the attenuation function $g(x, \xi)$ given by Eq. (34).

The numerical solution of the equilibrium Eqs. (32b, c) is found using a finite difference method. The numerical results reported are obtained for $N=800$ intervals in the beam domain. No significant differences are encountered for $N>800$. The finite difference method is applied using a standard discrete approximation of the differential operators and a standard trapezoidal approximation of the integrals in Eqs. (32b, c), and no difficulties are encountered in enforcing the B.C., as coincide with those of classical local theory; it can be implemented by any user, also by those not familiar with more involved and specific numerical methods as, for instance, the finite element method. The proposed finite difference solution involves separate local and non-local stiffness matrices, which correspond to the discretized differential operator and scalar integral, respectively. This means that, when implementing sensitivity analyses for varying local or non-local parameters, the local matrix or the non-local matrix only shall be updated.

\subsection{Numerical results}

Epoxy micro-beams with the following material properties are considered: Young's modulus $E=1.40 \mathrm{GPa}$, Poisson's coefficient $v=0.35$. The non-local parameters $C$ and $l$ in Eq. (34) are set on a theoretical basis, in order to enhance non-local effects and assess how they affect the static response. Specifically, $C=10^{11} \mathrm{Nm}^{-6}$ and different values of the internal length $l$ are considered.

\subsubsection{Simply-supported beam}

A simply-supported epoxy micro-beam with the following geometrical properties is considered: $L=300 \mu \mathrm{m}, h=L / 20=15 \mu \mathrm{m}, b=30 \mu \mathrm{m}$. A uniformly distributed load $p=1 \mathrm{Nm}^{-1}$ is assumed.

Figures 4 and 5 illustrate the dimensionless deflection $v(x) / L$ and rotation $\varphi(x)$ versus the non-dimensional location $x / L$, for different values of the internal length $l$. For comparison, the dimensionless classical local TM beam response is also reported. It is apparent 


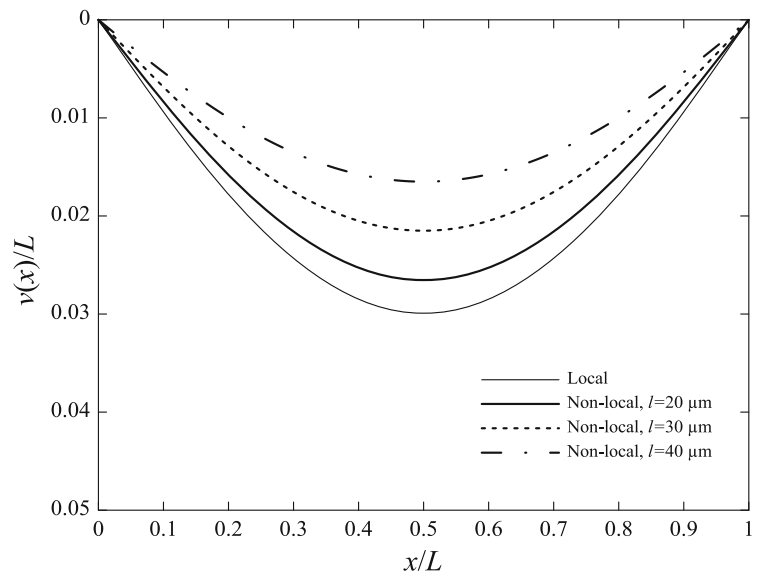

Fig. 4 Simply-supported beam: non-local and local dimensionless deflection for different $l$ (internal length)

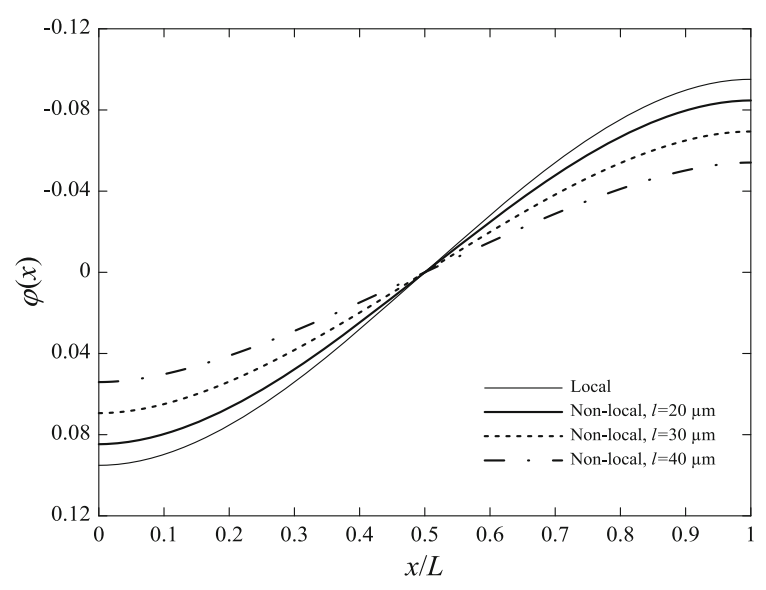

Fig. 5 Simply-supported beam: non-local and local rotation for different internal lengths $l$

that the non-local response is generally smaller than the classical local TM beam response. This behavior can be explained considering that the elastic longrange interactions provide additional stiffness with respect to the local terms in Eqs. (32b, c) and, since such terms coincide with the classical local TM beam terms $\left[\beta_{1}=1\right.$ has been set in Eq. (4)], the additional stiffness yields a non-local response that is stiffer than the classical local TM response. It can be also noted that the larger is the internal length $l$, the smaller is the non-local response: a larger internal length $l$ corresponds indeed to a larger amount of mutually interacting non-adjacent beam segments, with a consequent stiffening of the solution.

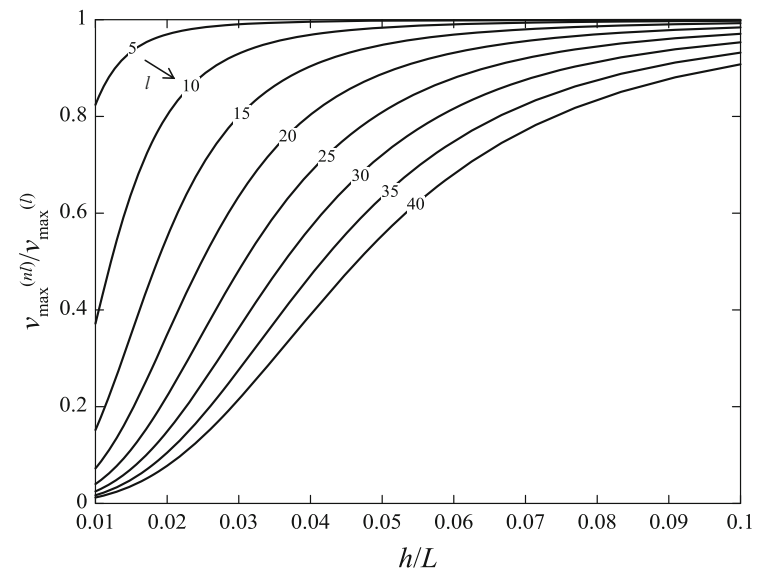

Fig. 6 Simply-supported beam: non-local to local maximum deflection ratio for $L=\operatorname{cost}$ (length), $b=\operatorname{cost}$ (width), variable $h$ (thickness) and different values of the internal length $l$ (from 5 to $40 \mu \mathrm{m})$

To have a better insight into the response of the proposed non-local beam model, the ratio of the midspan maximum non-local deflection to the mid-span maximum local deflection, $v_{\max }^{(n l)} / v_{\max }^{(l)}$, versus $h /$ $L$ (thickness-to-length ratio) is reported in Fig. 6, for fixed values of $L$ and $b$, i.e. $L=300 \mu \mathrm{m}$, $b=30 \mu \mathrm{m}$, different values of thickness $h$ and internal length $l$ (from 5 to $40 \mu \mathrm{m}$ ). It is seen that, for a given value of $l$, the smaller is $h$, the smaller is the non-local deflection, i.e. more significant are the non-local effects and, consequently, the deviation from the corresponding classical local TM response. To explain this behavior it is noticed that, if one considers the classical local TM beam without longrange interactions, for $L=$ cost and $b=$ cost the deformability increases with decreasing thickness $h$. As the beam undergoes deformation, the relative motion between non-adjacent beam segments activates the elastic long-range interactions, and their magnitude, for a given distance between two beam segments, depends on the magnitude of the relative motion [see Eqs. (11), (12), (13) and (14)]. For this reason, it is evident that the "weight" of the non-local terms shall increase as the deformability of beam increases, with the consequent stiffening effect with respect to the classical local TM beam response observed in Fig. 6, for decreasing thickness $h$. It can be also noted that larger deviations from the classical local response are encountered, as expected, for increasing values of the internal length $l$. It is quite 


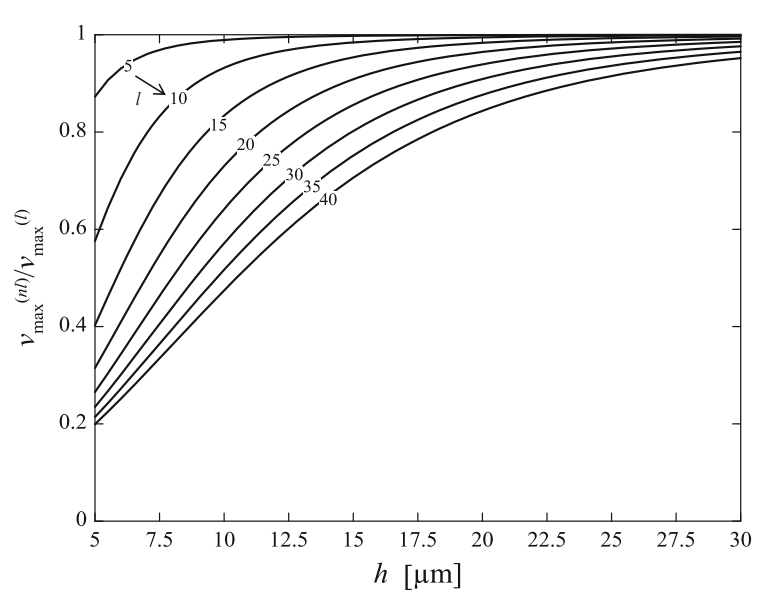

Fig. 7 Simply-supported beam: non-local to local maximum deflection ratio for $L / h=$ cost (length to thickness ratio), $b /$ $h=$ cost (width to thickness ratio), variable $h$ (thickness) and different values of the internal length $l$ (from 5 to $40 \mu \mathrm{m}$ )

interesting to point out that the stiffening effects shown in Fig. 6, for $L=$ cost and $b=$ cost and decreasing $h$ are predicted by other non-local theories [27] and are considered in agreement with experimental evidence on small-size effects in many materials [12, 13].

Further insight into the behavior of the proposed non-local beam model is provided by Fig. 7, that shows the ratio of the mid-span maximum non-local deflection to the mid-span maximum local deflection, $v_{\max }^{(n l)} / v_{\max }^{(l)}$, versus thickness $h$, for fixed values of the ratios $L / h=10, b / h=2$, and different values of the internal length $l$ (from 5 to $40 \mu \mathrm{m}$ ). As in the previous case, it is seen that the smaller is $h$ (i.e. the smaller are the overall dimensions of the beam being $L / h=$ cost), the smaller is the non-local deflection, i.e. more significant are the non-local effects. Such a behavior, for a given value of the internal length $l$, can be expected in consideration of the fact that $L$ decreases with $h(L / h=\mathrm{cost})$. As the beam becomes shorter while the internal length $l$ is fixed, each beam segment interacts with a relatively increasing number of beam segments (i.e., relatively to the total number of interacting beam segments) and, as a consequence, the "weight" of the non-local terms does increase with respect to that of the local ones. It can be also noted that larger deviations from the classical local response are encountered, as in Fig. 6, for increasing values of the internal length $l$. It is quite interesting to point out that the stiffening effects shown in Fig. 7, for $L /$ $h=$ cost, $b / h=$ cost, and decreasing $h$ are predicted

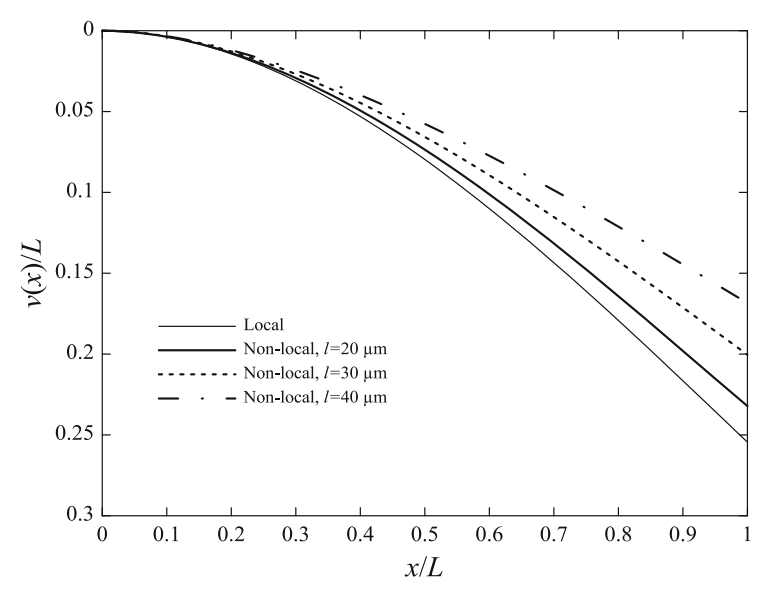

Fig. 8 Cantilever beam: non-local and local dimensionless deflection for different $l$ (internal length)

by other non-local theories [25] and are generally considered in agreement with experimental evidence on small-size stiffening effects in several materials $[12,13]$.

\subsubsection{Cantilever beam}

A cantilever epoxy micro-beam with the following parameters is considered: $L=300 \mu \mathrm{m}, \quad h=L /$ $20=15 \mu \mathrm{m}, b=30 \mu \mathrm{m}$. A tip load $P=100 \mu \mathrm{N}$ is assumed. Figure 8 through Fig. 11 show: (i) the dimensionless deflection $v(x) / L$ (Fig. 8) and rotation $\varphi(x)$ (Fig. 9) versus the non-dimensional location $x / L$, for different values of the internal length $l$; (ii) the ratio of the tip maximum non-local deflection to the tip maximum local deflection, $v_{\max }^{(n l)} / v_{\max }^{(l)}$, versus $h /$ $L$ (Fig. 10), for fixed values of $L$ and $b$, i.e. $L=300 \mu \mathrm{m}, b=30 \mu \mathrm{m}$, and different values of the internal length $l$ (from 5 to $40 \mu \mathrm{m}$ ); (iii) the ratio of the tip maximum non-local deflection to the tip maximum local deflection, $v_{\max }^{(n l)} / v_{\max }^{(l)}$, versus $h$ (Fig. 11), for fixed values of the ratio $L / h=10, b / h=2$, and different values of the internal length $l$ (from 5 to $40 \mu \mathrm{m}$ ). All the results appear in agreement with those obtained for a simply-supported beam. They can be explained based upon the same reasoning and, for this, further comments are omitted for brevity. It is only worth remarking that similar behaviors are predicted by alternative non-local theories [25, 27] and are generally considered in agreement with small-size stiffening effects in many materials $[12,13]$. 


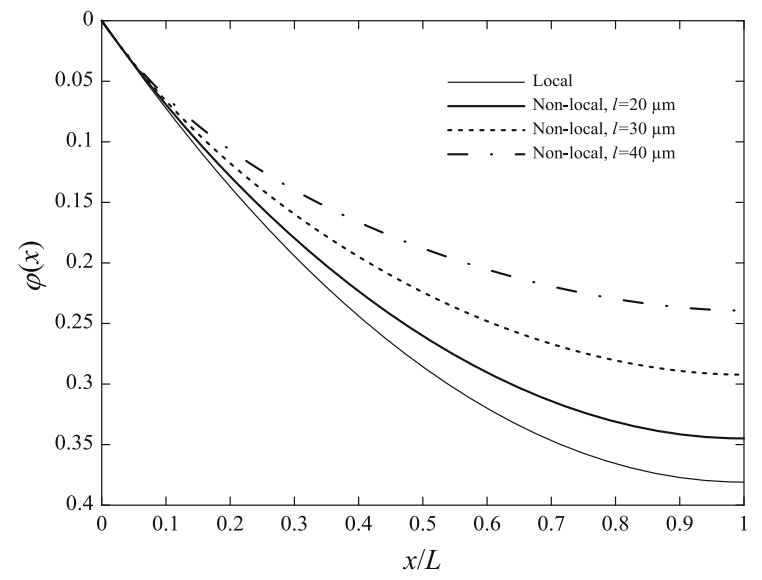

Fig. 9 Cantilever beam: non-local and local rotation for different $l$ (internal length)

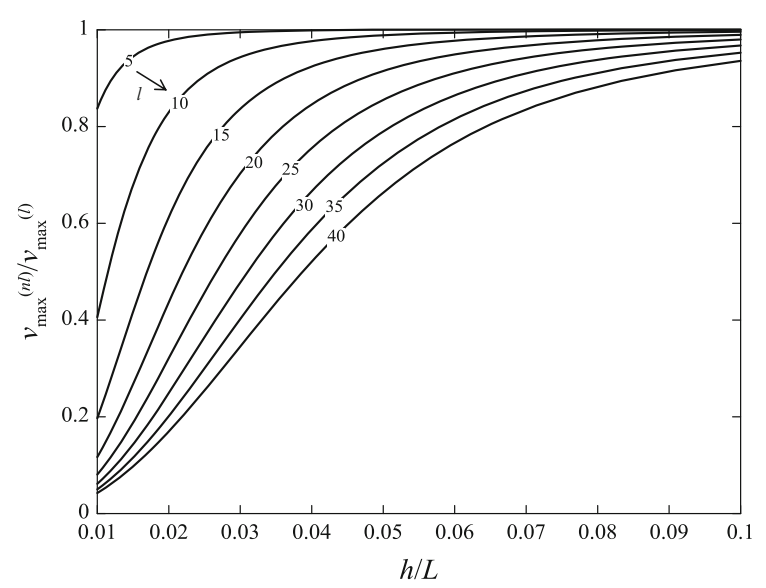

Fig. 10 Cantilever beam: non-local to local maximum deflection ratio for $L=$ cost (length), $b=$ cost (width), variable $h$ (thickness) and different values of the internal length $l$ (from 5 to $40 \mu \mathrm{m})$

\subsection{Comparison with experimental results}

Lam et al. [12] have reported the experimental response of a cantilever epoxy micro-beam with a rectangular cross section, subjected to a tip static load $P$. They have considered the following parameters [12, 36]: $b=0.235 \mathrm{~mm}, E=1.44 \mathrm{GPa}, \mathrm{v}=0.38$ (Poisson's coefficient), $P=300 \mu \mathrm{N}$. The response has been measured for a fixed ratio $h / L=0.1$ and four different thickness values $h$ : 20, 38, 75 and $115 \mu \mathrm{m}$. The results reported by Lam et al. [12] show that the experimental bending rigidity of the beam increases as the thickness decreases. This stiffening effect is not predicted by the

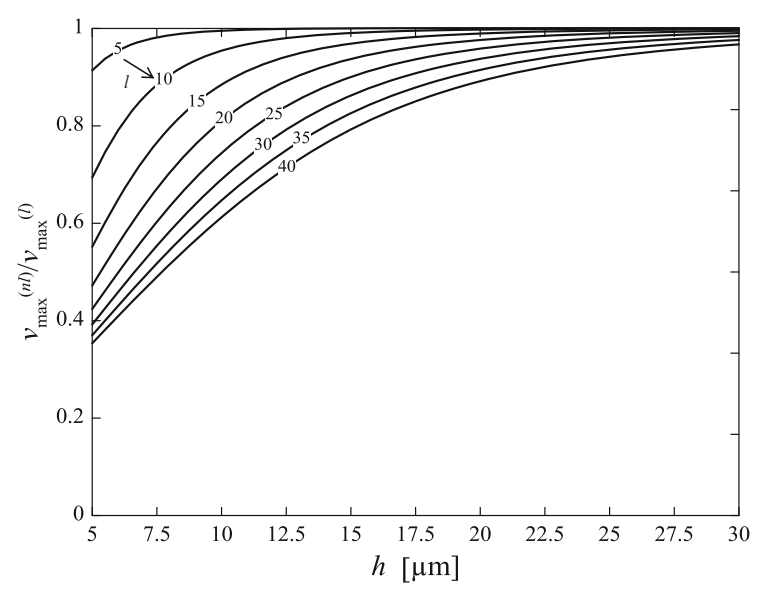

Fig. 11 Cantilever beam: non-local to local maximum deflection ratio for $L / h=$ cost (length to thickness ratio), $b / h=$ cost (width to thickness ratio), variable $h$ (thickness) and different values of the internal length $l$ (from 5 to $40 \mu \mathrm{m}$ )

classical EB beam theory, where the bending rigidity is constant when the ratio $h / L$ is held constant. It is now of interest to assess if, instead, it can be captured by the proposed non-local beam model.

For this purpose, the parameters/functions defining the proposed non-local beam model are selected as specified above, say: (i) $\beta_{1}=1$ in Eq. (4) for the local terms in Eqs. (32b, c), i.e. the local stiffness coincides with the stiffness of the classical TM theory; in this manner, the sought non-local solution will be stiffer than the local solution, consistently with the experimental behavior reported by Lam et al. [12]; (ii) the attenuation functions $g_{\varphi}(x, \xi)$ and $g_{z}(x, \xi)$ governing pure bending and pure shear non-local effects are given the exponential form (34). Being $\beta_{1}=1$ and due to the exponential form (34) of the attenuation functions, the sought non-local solution will revert to the classical local TM beam solution as $l \rightarrow 0$, as already pointed out in Sect. 5 .

Under these assumptions, the unknown parameters left are $C$ and $l$ in Eq. (34). To determine these parameters an error minimization procedure is pursued, based on the experimental data reported in Fig. 12 of the paper by Lam et al. [12]. They describe the ratio of the experimental bending rigidity to the classical bending rigidity of the EB beam theory, computed by Lam and coworkers as the ratio of the tip deflection of the classical EB beam theory $v^{(l)}(L)$ to the experimental tip deflection $v_{e x}(L)$ (see pag. 1503 of the paper by Lam et al. [12]), i.e. as 


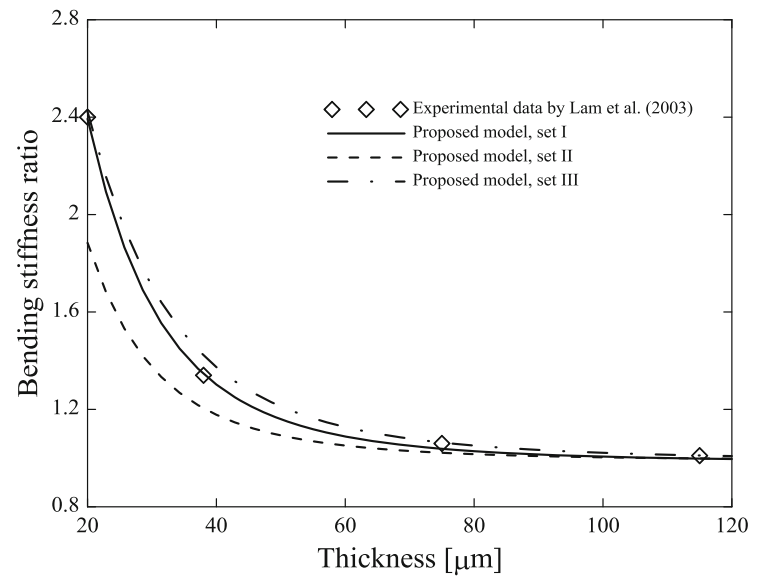

Fig. 12 Cantilever beam: bending stiffness ratio predicted by the proposed model for a varying thickness versus experimental data by Lam et al. [12]

$R_{e x}=\frac{v^{(l)}(L)}{v_{e x}(L)}$.

Therefore, in the error minimization procedure the sought values of $C$ and $l$ are computed as those that minimize, for the different thicknesses $(20,38,75$ and $115 \mu \mathrm{m})$ in Fig. 12 of the paper by Lam et al. [12], the squared difference between $R_{e x}$, Eq. (35), and

$R_{t h}=\frac{v^{(l)}(L)}{v(L)}$,

where $v(L)$ is the tip deflection predicted by the proposed non-local beam model. As in Sect. 5, the latter is computed by a finite difference approximation, with $N=800$ intervals in the beam domain. For completeness it shall be also noted that, in Fig. 12 of the paper by Lam et al. [12], a few experimental data are reported for each thickness $(20,38,75$ and 115 $\mu \mathrm{m})$. However since they appear very close, for each thickness their average value will be assumed as reference value in the error minimization procedure.

The results obtained in this manner are reported in Fig. 12. It can be seen that a very good agreement between the experimental bending rigidity ratios and those predicted by the proposed non-local beam model is attained for $C=3.17 \cdot 10^{11} \mathrm{Nm}^{-6}$ and $l=30 \mu \mathrm{m}$ in Eq. (34). Figure 12 reports the results obtained also for two alternative sets of $C, l$ (Set II: $C=$ $1.8 \cdot 10^{11} \mathrm{Nm}^{-6}, \quad l=30 \mu \mathrm{m}$; Set $\quad$ III: $C=$ $1.8 \cdot 10^{11} \mathrm{Nm}^{-6}, l=40 \mu \mathrm{m}$ ), in order to show that
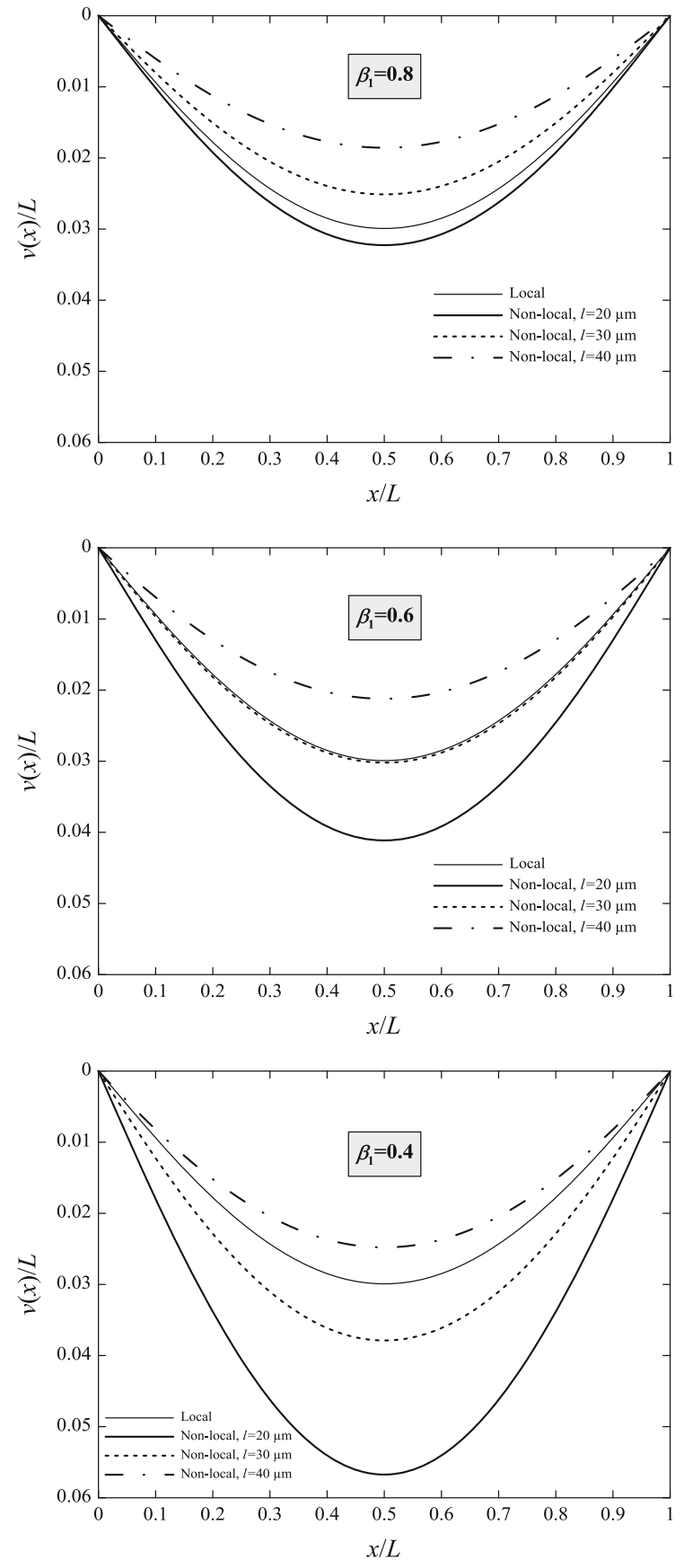

Fig. 13 Simply-supported beam: non-local and local dimensionless deflection for different $\beta_{1}$ in Eq. (4) and $l$ (internal length)

even for different sets of parameters, the qualitative behavior of the proposed non-local beam model still reflects the experimental data trend. 


\section{Conclusions}

A displacement-based non-local TM beam model has been illustrated. The key idea involves modeling nonlocal effects as elastic long-range volume forces/moments exchanged by non-adjacent beam segments, that contribute to the equilibrium of any beam segment along with the classical stress resultants. The longrange volume forces/moments have been built as linearly-elastic terms that depend, through appropriate attenuation functions, on the product of the volumes of the interacting beam segments and on generalized measures of their relative motion based on the pure deformation modes of the beam, to ensure invariance with respect to rigid body motion. This modeling has allowed a meaningful mechanical interpretation of the long-range interactions as resulting from three springlike connections acting in parallel, which account separately for pure axial, pure bending and pure shear modes.

The variational consistency of the model has been proved by deriving the beam equilibrium equations along with the pertinent B.C. based on a total elastic potential energy involving local and non-local contributions. The mechanical B.C. have been derived in the same form of the classical local theory. These aspects allow a straightforward implementation of classical solution methods, such as the finite difference method or the finite element method.

A certain number of parameters/functions shall be set in the proposed non-local beam model, concerning the local terms, [see $\beta_{1}$ in Eq. (4)] as well as non-local terms, namely the attenuation functions $\left[g_{x}(x, \xi)\right.$, $\left.g_{\varphi}(x, \xi), g_{z}(x, \xi)\right]$ with the related parameters. In practical applications, they shall be generally determined via an optimization procedure, where a fitting to experimental data is sought. It is worth remarking that a similar procedure is not necessary in the proposed non-local beam model only, but is necessary in all classical non-local theories involving an enriched continuum with additional non-local terms. Depending on the formulation, the non-local terms always involve a number of unknown parameters/functions that only experimental evidence may allow to select. For instance, examples of enriched continua with five additional parameters for non-local terms exist in the literature $[12,50]$, or non-local beam models with different potential choices of the attenuation functions $[49,51]$. Obviously, any optimization procedure to fit experimental evidence shall be generally preceded by numerical simulations, that may serve to determine the expected order of magnitude of the non-local terms, with respect to the local ones.

Numerical results, presented in Sect. 5 for a variety of geometrical parameters, have shown stiffening effects with respect to the classical local TM beam solution in agreement with the behavior predicted by alternative non-local theories [12, 25, 27, 36]. It has been also seen that the proposed non-local beam model captures very satisfactorily the experimental small-size stiffening effects reported by Lam et al. [12], for a cantilever epoxy micro-beam subjected to a tip static load. In all these applications, $\beta_{1}=1$ has been set in Eq. (4) for the local terms in Eqs. (32b, c) and exponential attenuation functions have been selected, depending on an internal length $l$ governing the spatial decay of non-local effects.

In the authors' opinion, the proposed non-local TM beam model involves some advantages, summarized as follows.

Due to the fact that non-local effects are modeled on a mechanical basis, specifically as elastic longrange force/moments counteracting the relative motion between couples of non-adjacent beam segments, the results provided by the proposed model are readily predictable. In general it can be stated that, because of the elastic long-range forces/moments counteracting the relative motion between beam segments, the nonlocal solution is generally stiffer than the local solution, i.e. the solution that would be obtained if only the local terms were considered in the equilibrium Eqs. (32). In particular:

(i) if $\beta_{1}=1$ is set in Eq. (4) for the local terms, as in the numerical applications of Sect. 5, the non-local solution will always be stiffer than the classical TM beam solution, because for $\beta_{1}=1$, the local terms in Eq. (4) coincide with the classical terms of the TM beam theory;

(ii) if $\beta_{1}<1$ is set in Eq. (4) for the local terms, the non-local solution will be either stiffer or softer than the classical TM beam solution. Because of the additional stiffness provided by the long-range interactions, the non-local solution will be stiffer than the local solution corresponding to the selected value $\beta_{1}<1$; however, because the latter is softer than the 
classical TM beam solution (that corresponds to $\beta_{1}=1$ ), the non-local solution will be either stiffer or softer than the classical TM beam solution depending on the amount of additional stiffness provided by the non-local terms, i.e. depending on the parameters of the attenuation functions $g_{x}(x, \xi), g_{\varphi}(x, \xi)$ and $g_{z}(x, \xi)$ (for the exponential functions considered in the paper such parameters are constant $C$ and internal length $l$ ). A pertinent example of this behavior is provided in Appendix 2. As a concluding comment in this respect, it is pointed out that the possibility of obtaining either stiffer or softer solutions with respect to the classical TM beam solution can be considered, in the authors' opinion, a quite useful feature of the proposed model, especially in view of the fact that experimental evidence on non-local effects is not yet fully available for all existing materials, and considering that most likely more complex materials will be produced in the future.

It is finally important to remark that the proposed mechanical description of non-local effects allows predicting how results may vary with beam geometry (e.g., for varying thickness with constant length and cross-section width, or for varying thickness with constant length-to-thickness and width-to-thickness ratios of the cross section), as discussed thoroughly in the comments on the numerical results reported in Sect. 5.

\section{Appendix 1}

In this Appendix, the derivation of identity (22) is presented. To this aim, first Eq. (22) is rewritten in the following form:

$$
\begin{aligned}
& \int_{0}^{L} \int_{0}^{L}\left[q_{x}(x, \xi) u(x)+q_{z}(x, \xi) v(x)+q_{\varphi \varphi}(x, \xi) \varphi(x)\right. \\
& \left.\quad+q_{\varphi z}(x, \xi) \varphi(x)\right] \mathrm{d} x \mathrm{~d} \xi \\
& =-\frac{1}{2} \int_{0}^{L} \int_{0}^{L}\left[q_{x}(x, \xi) \eta(x, \xi)+q_{\varphi \varphi}(x, \xi) \theta(x, \xi)\right. \\
& \left.\quad+q_{\varphi z}(x, \xi) \psi(x, \xi)\right] \mathrm{d} x \mathrm{~d} \xi
\end{aligned}
$$

where the definitions of the vectors $\mathbf{u}(x), \tilde{\mathbf{q}}(x, \xi), \mathbf{e}(x, \xi)$ and $\mathbf{q}(x, \xi)$ have been introduced [see Eqs. (20a, c), (23a, b)]. Then, Eq. (37) holds if the following identities are fulfilled:

$$
\begin{aligned}
& \int_{0}^{L} \int_{0}^{L} q_{x}(x, \xi) u(x) \mathrm{d} x \mathrm{~d} \xi \\
& =-\frac{1}{2} \int_{0}^{L} \int_{0}^{L} q_{x}(x, \xi) \eta(x, \xi) \mathrm{d} x \mathrm{~d} \xi ; \\
& \int_{0}^{L} \int_{0}^{L} q_{\varphi \varphi}(x, \xi) \varphi(x) \mathrm{d} x \mathrm{~d} \xi \\
& =-\frac{1}{2} \int_{0}^{L} \int_{0}^{L} q_{\varphi \varphi}(x, \xi) \theta(x, \xi) \mathrm{d} x \mathrm{~d} \xi \\
& \int_{0}^{L} \int_{0}^{L}\left[q_{z}(x, \xi) v(x)+q_{\varphi z}(x, \xi) \varphi(x)\right] \mathrm{d} x \mathrm{~d} \xi \\
& =-\frac{1}{2} \int_{0}^{L} \int_{0}^{L} q_{\varphi z}(x, \xi) \psi(x, \xi) \mathrm{d} x \mathrm{~d} \xi .
\end{aligned}
$$

To prove Eq. (38), it is observed that due to the symmetry of $g_{x}(x, \xi)$, one may write:

$$
\begin{aligned}
\int_{0}^{L} & \int_{0}^{L} q_{x}(x, \xi) u(x) \mathrm{d} x \mathrm{~d} \xi \\
= & -\int_{0}^{L} \int_{0}^{L} q_{x}(x, \xi) u(\xi) \mathrm{d} x \mathrm{~d} \xi .
\end{aligned}
$$

Adding to both sides of Eq. (41) the term $\int_{0}^{L} \int_{0}^{L} q_{x}(x, \xi) u(x) \mathrm{d} x \mathrm{~d} \xi$, Eq. (38) can be obtained.

Equation (39) can be derived following a similar reasoning. Indeed, the symmetry of $g_{\varphi}(x, \xi)$ allows us to write:

$$
\begin{aligned}
& \int_{0}^{L} \int_{0}^{L} q_{\varphi \varphi}(x, \xi) \varphi(x) \mathrm{dxd} \xi \\
& =-\int_{0}^{L} \int_{0}^{L} q_{\varphi \varphi}(x, \xi) \varphi(\xi) \mathrm{d} x \mathrm{~d} \xi .
\end{aligned}
$$

Then, adding to both sides of the previous equation the term $\int_{0}^{L} \int_{0}^{L} q_{\varphi}(x, \xi) \varphi(x) \mathrm{d} x \mathrm{~d} \xi$, Eq. (39) is readily obtained.

Equation (40) can be split in the following two identities

$$
\begin{aligned}
& \int_{0}^{L} \int_{0}^{L} q_{z}(x, \xi) v(x) \mathrm{d} x \mathrm{~d} \xi \\
& =-\frac{1}{2} \int_{0}^{L} \int_{0}^{L} q_{\varphi z}(x, \xi)\left[2\left(\frac{v(\xi)-v(x)}{\xi-x}\right)\right] \mathrm{d} x \mathrm{~d} \xi
\end{aligned}
$$




$$
\begin{aligned}
\int_{0}^{L} & \int_{0}^{L} q_{\varphi z}(x, \xi) \varphi(x) \mathrm{d} x \mathrm{~d} \xi \\
\quad= & \frac{1}{2} \int_{0}^{L} \int_{0}^{L} q_{\varphi z}(x, \xi)[\varphi(x)+\varphi(\xi)] \mathrm{d} x \mathrm{~d} \xi .
\end{aligned}
$$

To prove Eq. (43), let us first substitute in this equation the definitions (13b) and (14b) of $q_{z}(x, \xi)$ and $q_{\varphi z}(x, \xi)$, respectively:

$$
\begin{aligned}
& \int_{0}^{L} \int_{0}^{L} 2 \frac{\operatorname{sgn}(\xi-x)}{|\xi-x|} g_{z}(x, \xi) \psi(x, \xi) v(x) \mathrm{d} x \mathrm{~d} \xi \\
& =-\frac{1}{2} \int_{0}^{L} \int_{0}^{L} g_{z}(x, \xi) \psi(x, \xi) \\
& \quad \times\left[2\left(\frac{v(\xi)-v(x)}{\xi-x}\right)\right] \mathrm{d} x \mathrm{~d} \xi .
\end{aligned}
$$

Due to the symmetry of the attenuation function $g_{z}(x, \xi)$, the following relationship holds:

$$
\begin{aligned}
& \int_{0}^{L} \int_{0}^{L} 2 \frac{\operatorname{sgn}(\xi-x)}{|\xi-x|} g_{z}(x, \xi) \\
& \quad \times\left[2\left(\frac{v(\xi)-v(x)}{\xi-x}\right)\right] v(x) \mathrm{d} x \mathrm{~d} \xi \\
& =-\int_{0}^{L} \int_{0}^{L} 2 \frac{\operatorname{sgn}(\xi-x)}{|\xi-x|} g_{z}(x, \xi) \\
& \quad \times\left[2\left(\frac{v(\xi)-v(x)}{\xi-x}\right)\right] v(\xi) \mathrm{d} x \mathrm{~d} \xi .
\end{aligned}
$$

Then, adding to both sides of Eq. (46) the integral on the 1.h.s. of this equation, the following identity is obtained:

$$
\begin{aligned}
& \int_{0}^{L} \int_{0}^{L} 2 \frac{\operatorname{sgn}(\xi-x)}{|\xi-x|} g_{z}(x, \xi)\left[2\left(\frac{v(\xi)-v(x)}{\xi-x}\right)\right] v(x) \mathrm{d} x \mathrm{~d} \xi \\
& =-\frac{1}{2} \int_{0}^{L} \int_{0}^{L} g_{z}(x, \xi)\left[2\left(\frac{v(\xi)-v(x)}{\xi-x}\right)\right] \\
& \quad \times 2\left(\frac{v(\xi)-v(x)}{\xi-x}\right) \mathrm{d} x \mathrm{~d} \xi .
\end{aligned}
$$

where $\operatorname{sgn}(\xi-x) /|\xi-x|=1 /(\xi-x)$ has been set on the r.h.s. Furthermore, the symmetry of the function $g_{z}(x, \xi)$ allows us to write:

$$
\begin{aligned}
& \int_{0}^{L} \int_{0}^{L} 2 \frac{\operatorname{sgn}(\xi-x)}{|\xi-x|} g_{z}(x, \xi)[\varphi(x) \\
& \quad+\varphi(\xi)] v(x) \mathrm{d} x \mathrm{~d} \xi \\
& =-\int_{0}^{L} \int_{0}^{L} 2 \frac{\operatorname{sgn}(\xi-x)}{|\xi-x|} g_{z}(x, \xi)[\varphi(x) \\
& \quad+\varphi(\xi)] v(\xi) \mathrm{d} x \mathrm{~d} \xi
\end{aligned}
$$

Then, adding to both sides of Eq. (48) the integral on the 1.h.s., yields:

$$
\begin{aligned}
& \int_{0}^{L} \int_{0}^{L} 2 \frac{\operatorname{sgn}(\xi-x)}{|\xi-x|} g_{z}(x, \xi)[\varphi(x)+\varphi(\xi)] v(x) \mathrm{d} x \mathrm{~d} \xi \\
& =-\frac{1}{2} \int_{0}^{L} \int_{0}^{L} g_{z}(x, \xi)[\varphi(x)+\varphi(\xi)] \\
& \quad \times 2\left(\frac{v(\xi)-v(x)}{\xi-x}\right) \mathrm{d} x \mathrm{~d} \xi .
\end{aligned}
$$

where, as in Eq. (47), $\operatorname{sgn}(\xi-x) /|\xi-x|=1 /(\xi-x)$ has been set on the r.h.s. Subtracting both sides of Eqs. (47) and (49) and recalling the definition (10) of $\psi(x, \xi)$, Eq. (43) is obtained.

Finally, to prove Eq. (44), it is observed that due to the symmetry of $g_{z}(x, \xi)$, the following identity holds:

$$
\begin{aligned}
& \int_{0}^{L} \int_{0}^{L} g_{z}(x, \xi) \psi(x, \xi) \varphi(x) \mathrm{d} x \mathrm{~d} \xi \\
& =\int_{0}^{L} \int_{0}^{L} g_{z}(x, \xi) \psi(x, \xi) \varphi(\xi) \mathrm{d} x \mathrm{~d} \xi .
\end{aligned}
$$

Then, adding the 1.h.s of Eq. (50) to both sides of the same equation and taking into account the definition (14b) of $q_{\varphi z}(x, \xi)$, Eq. (44) is obtained.

\section{Appendix 2}

The purpose of this Appendix is to briefly illustrate the behavior of the proposed non-local beam model when $\beta_{1}<1$ is set in Eq. (4) for the local terms in Eqs. (32b, c). This is of interest to show, as discussed in the Conclusions, that the proposed non-local beam model 
is potentially capable of predicting non-local solutions that may be either stiffer or softer with respect to the classical local TM beam response.

A simply-supported beam is considered. $\mathrm{Pa}$ rameters and loading conditions are taken as in Sect. 5.1.1, while $\beta_{1}$ in Eq. (4) is given the following values: $\beta_{1}=0.4 ; 0.6 ; 0.8$. The dimensionless deflection $v(x) / L$ versus the non-dimensional location $x / L$ is reported in Fig. 13, for different values of the internal length $l$. For comparison, the classical local TM beam response, corresponding to $\beta_{1}=1$ and no long-range resultants in Eqs. (32b, c), is also reported.

For a given value $\beta_{1}$, it is seen that the non-local response may be either stiffer or softer than the classical local TM beam response, depending on the internal length $l$. This behavior can be explained considering that, for $\beta_{1}<1$, the solution provided by the local terms only in Eqs. (32b, c), i.e. without longrange resultants, is obviously softer than the classical local TM beam response [corresponding to $\beta_{1}=1$ and no long-range resultants in Eqs. $(32 \mathrm{~b}, \mathrm{c})]$. The longrange resultants provide additional stiffness with respect to that of the local terms in Eqs. $(32 b, c)$, but such additional stiffness may not be enough to make the non-local response stiffer than the classical local TM beam response. In particular, Fig. 13 shows that the non-local response becomes progressively stiffer with increasing $l$, consistently with the fact a larger internal length $l$ corresponds indeed to a larger amount of mutually interacting non-adjacent beam segments, with a consequent stiffening (see also comments on Figs. 4 and 5). The comments above explain also the fact that, in Fig. 13, a softer non-local response is obtained as parameter $\beta_{1}$ decreases, for a given internal length $l$.

The rotation response is in accordance with the deflection response in Fig. 13 and pertinent results are not reported for conciseness. Likewise, results for a cantilever beam subjected to a tip load agree with those for the simply-supported beam and are omitted.

\section{References}

1. Eringen AC (1972) Linear theory of nonlocal elasticity and dispersion of plane waves. Int J Eng Sci 10:425-435

2. Eringen AC (1983) On differential equations of nonlocal elasticity and solutions of screw dislocation and surface waves. J Appl Phys 54:4703-4710
3. Aifantis EC (1999) Gradient deformation models at nano, micro, and macroscales. J Eng Mater Technol-Trans ASME 121:189-202

4. Chang CS, Askes H, Sluys LJ (2002) Higher-order strain/ higher-order stress gradient models derived from a discrete microstructure, with application to fracture. Eng Fract Mech 69:1907-1924

5. Silling SA, Zimmermann M, Abeyaratne R (2003) Deformation of a peridynamic bar. J Elast 73:173-190

6. Cosserat E, Cosserat F (1909) Théorie des Corps Déformables. Hermann, Paris

7. Nowacki W (1986) Theory of Asymmetric Elasticity. Polish Scientific Publishers, Warsaw

8. Kröner E (1963) On the physical reality of torque stresses in continuum mechanics. Int J Eng Sci 1(2):261-262

9. Lakes RS (1991) Experimental micro mechanics methods for conventional and negative Poisson's ratio cellular solids as Cosserat continua. J Eng Mater Technol 113:148-155

10. Qian D, Wagner GJ, Liu WK, Yu MF, Ruoff RS (2002) Mechanics of carbon nanotubes. Appl Mech Rev 55(6):495-533

11. Wang LF, Hu HY (2005) Flexural wave propagation in singlewalled carbon nanotube. Phys Rev B 71:195412-195418

12. Lam DCC, Yang F, Chong ACM, Wang J, Tong P (2003) Experiments and theory in strain gradient elasticity. J Mech Phys Solids 51:1477-1508

13. McFarland AW, Colton JS (2005) Role of material microstructure in plate stiffness with relevance to microcantilever sensors. J Micromech Microeng 15:1060-1067

14. Tang PY (1983) Interpretation of bend strength increase of graphite by the couple stress theory. Comput Struct 16:45-49

15. Poole WJ, Ashby MF, Fleck NA (1996) Micro-hardness of annealed and work-hardened copper polycrystals. Scripta Mater 34(4):559-564

16. Zhang YQ, Liu GR, Xie XY (2005) Free transverse vibrations of double-walled carbon nanotubes using a theory of nonlocal elasticity. Phys Rev B 71:195404

17. Xu M (2006) Free transverse vibrations of nano-to-micron scale beams. Proc R Soc A 462:2977-2995

18. Wang Q, Varadan VK (2006) Vibration of carbon nanotubes studied using nonlocal continuum mechanics. Smart Mater Struct 15:659-666

19. Lu P, Lee HP, Lu C, Zhang PQ (2007) Application of nonlocal beam models for carbon nanotubes. Int J Solids Struct 44:5289-5300

20. Reddy JN (2007) Nonlocal theories for bending, buckling and vibration of beams. Int J Eng Sci 45:288-307

21. Aydogdu M (2009) A general nonlocal beam theory: its application to nanobeam bending, buckling and vibration. Physica E 41:1651-1655

22. Kong S, Zhou S, Nie Z, Wang K (2008) The size-dependent natural frequency of Bernoulli-Euler micro-beams. Int $\mathbf{J}$ Eng Sci 46:427-437

23. Zhang YY, Wang CM, Challamel N (2010) Bending, buckling and vibration of micro/nanobeams by hybrid nonlocal beam model. J Eng Mech 136(5):562-574

24. Wang B, Zhao J, Zhou S (2010) A micro scale Timoshenko beam model based on strain gradient elasticity theory. Eur $\mathbf{J}$ Mech A/Solids 29:591-599

25. Ma HM, Gao X-L, Reddy JN (2008) A microstructure-dependent Timoshenko beam model based on a modified couple stress theory. J Mech Phys Solids 56:3379-3391 
26. Pradhan SC (2012) Nonlocal finite element analysis and small scale effects of CNTs with Timoshenko beam theory. Finite Elem Anal Des 50:8-20

27. Yang Y, Lim CW (2012) Non-classical stiffness strengthening size effects for free vibration of a nonlocal nanostructure. Int J Mech Sci 54:57-68

28. Lim CW (2010) On the truth of nanoscale for nanobeams based on nonlocal elastic stress field theory: equilibrium, governing equation and static deflection. Appl Math Mech 31(1):37-54

29. Lim CW, Yang Y (2010) New predictions of size-dependent nanoscale based on nonlocal elasticity for wave propagation in carbon nanotubes. J Comput Theor Nanosci 7(6):988-995

30. Peddieson J, Buchanan GR, McNitt RP (2003) Application of nonlocal continuum models to nanotechnology. Int J Eng Sci 41:305-312

31. Wang Q, Shindo Y (2006) Nonlocal continuum models for carbon nanotubes subjected to static loading. J Mech Mater Struct 1:663-680

32. Civalek Ö, Demir Ç (2011) Bending analysis of microtubules using nonlocal Euler-Bernoulli beam theory. Appl Math Model 35:2053-2067

33. Wang Q, Liew KM (2007) Application of nonlocal continuum mechanics to static analysis of micro- and nanostructures. Phys Lett A 363:236-242

34. Wang CM, Kitipornchai S, Lim CW, Eisenberger M (2008) Beam bending solutions based on nonlocal Timoshenko beam theory. J Eng Mech 134:475-481

35. Challamel N, Wang CM (2008) The small length scale effect for a non-local cantilever beam: a paradox solved. Nanotechnology 19:345703

36. Park SK, Gao X-L (2006) Bernoulli-Euler beam model based on a modified couple stress theory. J Micromech Microeng 16:2355-2359

37. Chen SH, Feng B (2011) Size effect in micro-scale cantilever beam bending. Acta Mech 219:291-307

38. Akgöz B, Civalek Ö (2012) Analysis of micro-sized beams for various boundary conditions based on the strain gradient elasticity theory. Arch Appl Mech 82:423-443
39. Di Paola M, Failla G, Sofi A, Zingales M (2011) A mechanically based approach to non-local beam theories. Int $\mathrm{J}$ Mech Sci 53:676-687

40. Di Paola M, Failla G, Sofi A, Zingales M (2012) On the vibrations of a mechanically based non-local beam model. Comput Mater Sci 64:278-282

41. Di Paola M, Failla G, Zingales M (2013) Non-local stiffness and damping models for shear-deformable beams. Eur $\mathbf{J}$ Mech A/Solid 40:69-83

42. Di Paola M, Failla G, Zingales M (2009) Physically-based approach to the mechanics of strong non-local linear elasticity theory. J Elast 97:103-130

43. Di Paola M, Pirrotta A, Zingales M (2010) Mechanicallybased approach to non-local elasticity: variational principles. Int J Solids Struct 47:539-548

44. Di Paola M, Failla G, Zingales M (2010) The mechanicallybased approach to $3 \mathrm{D}$ non-local linear elasticity theory: long-range central interactions. Int $\mathrm{J}$ Solids Struct 47:2347-2358

45. Di Paola M, Sofi A, Zingales M (2011) Stochastic analysis of one-dimensional heterogeneous solids with long-range interactions. Int J Multiscale Comput Eng 9(4):379-394

46. Failla G, Santini A, Zingales M (2010) Solution strategies for $1 \mathrm{D}$ elastic continuum with long-range interactions: smooth and fractional decay. Mech Res Commun 37:13-21

47. Fuchs MB (1991) Unimodal beam elements. Int J Solids Struct 27(5):533-545

48. Fuchs MB (1997) Unimodal formulation of the analysis and design problems for framed structures. Comput Struct 63(4):739-747

49. Friswell MI, Adhikari S, Lei Y (2007) Non-local finite element analysis of damped beams. Int $\mathbf{J}$ Solids Struct 44:7564-7576

50. Mindlin RD (1965) Second gradient of strain and surface tension in linear elasticity. Int J Solids Struct 1:417-438

51. Lei Y, Friswell MI, Adhikari S (2006) A Galerkin method for distributed systems with non-local damping. Int J Solids Struct 43:3381-3400 